\title{
Holes Localized on a Skyrmion in a Doped Antiferromagnet on the Honeycomb Lattice: Symmetry Analysis
}

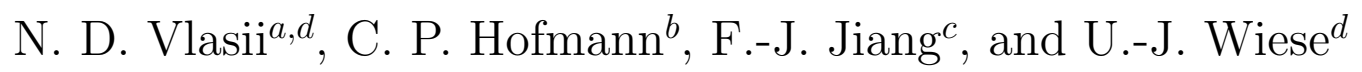 \\ ${ }^{a}$ Bogolyubov Institute for Theoretical Physics, \\ National Academy of Sciences of Ukraine, \\ 14-b Metrologichna Str., Kyiv, 03680, Ukraine \\ ${ }^{b}$ Facultad de Ciencias, Universidad de Colima \\ Colima C.P. 28045, Mexico \\ ${ }^{c}$ Department of Physics, National Taiwan Normal University \\ 88, Sec. 4, Ting-Chou Rd., Taipei 116, Taiwan \\ ${ }^{d}$ Albert Einstein Center for Fundamental Physics \\ Institute for Theoretical Physics, Bern University \\ Sidlerstrasse 5, CH-3012 Bern, Switzerland
}

October 2, 2014

\begin{abstract}
Using the low-energy effective field theory for hole-doped antiferromagnets on the honeycomb lattice, we study the localization of holes on Skyrmions, as a potential mechanism for the preformation of Cooper pairs. In contrast to the square lattice case, for the standard radial profile of the Skyrmion on the honeycomb lattice, only holes residing in one of the two hole pockets can get localized. This differs qualitatively from hole pairs bound by magnon exchange, which is most attractive between holes residing in different momentum space pockets. On the honeycomb lattice, magnon exchange unambiguously leads to $f$-wave pairing, which is also
\end{abstract}


observed experimentally. Using the collective-mode quantization of the Skyrmion, we determine the quantum numbers of the localized hole pairs. Again, $f$-wave symmetry is possible, but other competing pairing symmetries cannot be ruled out.

\section{Introduction}

Understanding the mechanism underlying high-temperature superconductivity has remained a major challenge in condensed matter physics. Since high-temperature cuprate superconductors are insulating antiferromagnets before doping, it is natural to also investigate their antiferromagnetic precursors. In particular, one may hope to identify potential mechanisms for Cooper pair preformation in the antiferromagnetic phase. While we do not necessarily expect to unravel the relevant mechanism in this way, it motivates a careful systematic study. In previous work we have investigated the interactions between holes in lightly doped antiferromagnets, using a systematic lowenergy effective field theory approach, both on the square [1] and on the honeycomb lattice [2].

The effective theory is formulated in terms of the staggered magnetization order parameter field, whose fluctuations correspond to spinwaves (magnons), and in terms of fermionic hole fields. This is in complete analogy to baryon chiral perturbation theory in particle physics, where the fluctuations in the chiral order parameter manifest themselves as pions, while baryons (protons and neutrons) are analogous to the doped holes 3 6]. Based on microscopic Hubbard and $t-J$ models, and using a systematic low-energy expansion, we have constructed effective field theories for magnons and holes, both on the square and on the honeycomb lattice [1, 2, 711]. In both cases, one-magnon exchange mediates weak attractive forces between doped holes. As doping is increased, antiferromagnetism is weakened, and ultimately disappears as a long-range order phenomenon, when one enters the superconducting phase. Still, intermediate-range antiferromagnetic correlations persist even in the superconductor, and it is interesting to ask which objects form when one is about to leave the antiferromagnetic phase. At the edge of this phase, the spin stiffness $\rho_{s}$ decreases and the energy $4 \pi \rho_{s}$ of Skyrmion excitations in the staggered magnetization order parameter is thus reduced. In addition, doped holes can gain energy when they localize on a topological Skyrmion defect. We have systematically investigated this phenomenon as a potential mechanism for Cooper pair preformation for antiferromagnets on the square lattice [12]. Interestingly, in this case, both one-magnon exchange and Skyrmion localization lead to bound states in the same symmetry channel. The role of Skyrmions in quantum antiferromagnets has been investigated in [13 31].

The main purpose of the present paper is to extend the study of hole localization on a Skyrmion to antiferromagnets on the honeycomb lattice, which underlies certain 
high-temperature superconductors, including the dehydrated version of $\mathrm{Na}_{2} \mathrm{CoO}_{2} \times$ $y \mathrm{H}_{2} \mathrm{O}$. In this case, experiments suggest that the pairing symmetry is $f$-wave [32]. $F$-wave pairing has also been found for other strongly correlated systems on the honeycomb lattice [33 35]. As we studied earlier [2], in contrast to the square lattice case, on the honeycomb lattice, leading order one-magnon exchange gives rise to longrange attraction only between holes residing in different hole pockets (characterized by the "flavors" $\alpha$ and $\beta$ ). As an unambiguous prediction of the effective theory, the binding occurs in the $f$-wave channel, and is thus indeed consistent with experiment [32]. As we will show here, unlike in the square lattice case, on the honeycomb lattice the symmetry channels favored by hole localization on Skyrmions are not in one-to-one correspondence with the symmetry channels resulting from one-magnon exchange. In particular, a Skyrmion with the standard radial profile can only localize holes residing in the $\alpha$-pocket, while an anti-Skyrmion can only localize $\beta$-holes. While $f$-wave symmetry still arises, other competing pairing symmetries are possible as well. Only detailed energetic considerations, which we leave for future work, can unambiguously decide which pairing mechanism is favored by Skyrmion localization. In this paper, we concentrate entirely on the systematic symmetry analysis of the various hole states localized on a Skyrmion.

The rest of the paper is organized as follows. In Section 2 we review the effective field theory formulation of antiferromagnetic magnons on the honeycomb lattice and discuss Skyrmions as classical solutions. We also comment on the Hopf term and on the collective modes of a rotating Skyrmion which we then quantize. In Section 3, the effective field theory description is extended by introducing holes doped into the system. Section 4 is devoted to the symmetry analysis of states of single holes as well as hole pairs of the same flavor, localized on a static or a rotating Skyrmion. While our main focus is the symmetry analysis, some simple energetic considerations are also discussed. While Section 6 contains our conclusions, the localization of two holes with different flavor on a Skyrmion is investigated in the Appendix.

\section{Skyrmions in Magnon Effective Field Theory}

In this section, we consider the effective description of magnons and Skyrmions, i.e., we restrict ourselves to the undoped honeycomb lattice antiferromagnet.

\subsection{Symmetries of the Effective Action}

Magnons are the Goldstone bosons which result from the spontaneously broken spin symmetry $S U(2)_{s} \rightarrow U(1)_{s}$. They are described by a 3-component unit-vector field $\vec{e}(x) \in S^{2}$, living in the coset space $S^{2}=S U(2)_{s} / U(1)_{s}$. The coordinate $x=\left(x_{1}, x_{2}, t\right)$ represents a point in $(2+1)$-dimensional Euclidean space-time, while the vector $\vec{e}(x)$ 
points into the direction of the local staggered magnetization, i.e., into the direction of the order parameter of the spontaneously broken spin rotation symmetry. The Euclidean effective action for the magnons, at leading order in the systematic derivative expansion, takes the form

$$
S[\vec{e}]=\int d^{2} x d t \frac{\rho_{s}}{2}\left(\partial_{i} \vec{e} \cdot \partial_{i} \vec{e}+\frac{1}{c^{2}} \partial_{t} \vec{e} \cdot \partial_{t} \vec{e}\right) .
$$

The low-energy couplings $\rho_{s}$ and $c$ are the spin stiffness and the spinwave velocity, respectively. The ground state of the system is described by a constant staggered magnetization vector which we choose to point in the 3-direction: $\vec{e}(x)=(0,0,1)$. The spin waves or magnons then correspond to small fluctuations around the vector $\vec{e}(x)=(0,0,1)$. Unlike for ferromagnetic magnons which follow a quadratic dispersion law, antiferromagnetic magnons obey a linear, i.e., "relativistic" dispersion relation. Note that the leading-order effective action for the magnons on the honeycomb lattice, Eq. (2.1), is identical with the one on the square lattice. In general, lattice anisotropies only start manifesting themselves at subleading orders.

Note that the $S U(2)_{s}$ spin symmetry corresponds to an internal symmetry, much like chiral symmetry in particle physics. Hence its unbroken $U(1)_{s}$ subgroup (which in particle physics corresponds to isospin) also represents an internal symmetry. In view of this analogy, transformations in the unbroken subgroup $U(1)_{s}$ are denoted by $I(\gamma)$. In the construction of the effective field theory, the spontaneously broken spin symmetry $S U(2)_{s} \rightarrow U(1)_{s}$ plays an essential role. In particular, global transformations in the unbroken subgroup $U(1)_{s}$ will be important. We parametrize the order parameter vector as

$$
\vec{e}(x)=(\sin \theta(x) \cos \varphi(x), \sin \theta(x) \sin \varphi(x), \cos \theta(x)) .
$$

Under the global transformations, this vector transforms as

$$
{ }^{I(\gamma)} \vec{e}(x)=(\sin \theta(x) \cos (\varphi(x)+\gamma), \sin \theta(x) \sin (\varphi(x)+\gamma), \cos \theta(x)) .
$$

Apart from the spontaneously broken spin symmetry $S U(2)_{s}$, the effective action exhibits further symmetries, both continuous and discrete. First of all, the leadingorder expression of the effective action, Eq. (2.1), is Poincaré invariant. Note that we are dealing with an accidental symmetry on the effective level, which is not shared by the underlying Hubbard or $t-J$ models. It only emerges at leading order of the effective action. The discrete symmetries are translations, rotations, and reflections of the underlying honeycomb lattice, which we have depicted in Fig. 1 with its two translation vectors $a_{1}$ and $a_{2}$. The displacements $D_{i}$ along these primitive lattice vectors leave the staggered magnetization invariant, such that the field $\vec{e}(x)$ transforms trivially,

$$
{ }^{D_{i}} \vec{e}(x)=\vec{e}(x) .
$$




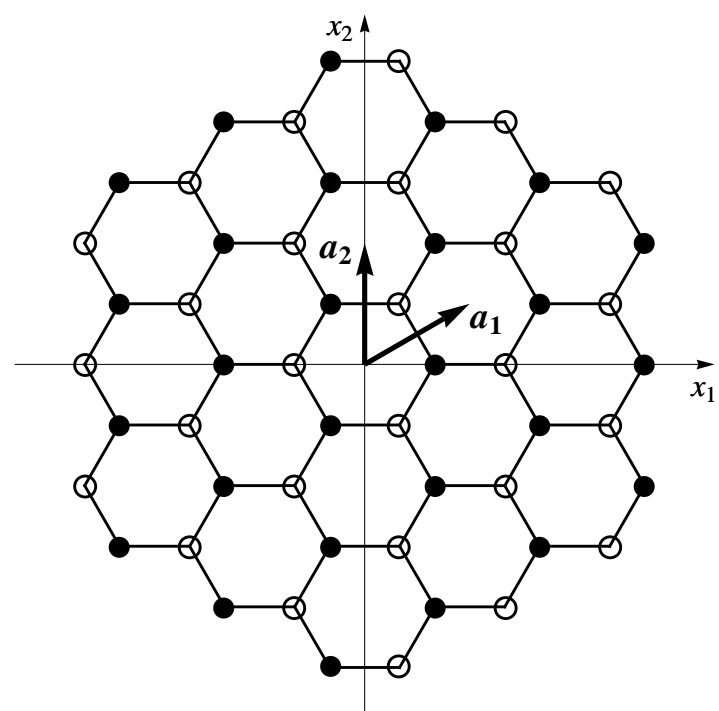

Figure 1: The bipartite non-Bravais honeycomb lattice consists of two triangular Bravais sublattices. The quantities $a_{1}$ and $a_{2}$ are the two independent primitive lattice vectors.

Rotations by 60 degrees around an axis located at the center of a hexagon act on the staggered magnetization vector as

$$
{ }^{O} \vec{e}(x)=-\vec{e}(O x)
$$

Note that, on the honeycomb lattice the discrete rotation $O$ by 60 degrees is spontaneously broken, while on the square lattice rotations by 90 degrees are not. It is convenient to also introduce the modified rotation symmetry, $O^{\prime}$, which is a combination of the simple rotation $O$ and the $S U(2)_{s}$ spin rotation $g=i \sigma_{2}$. In contrast to $O$, the combined symmetry $O^{\prime}$ is not spontaneously broken. Under $O^{\prime}$ the staggered magnetization vector transforms as

$$
{ }^{O^{\prime}} \vec{e}(x)=\left(e_{1}(O x),-e_{2}(O x), e_{3}(O x)\right) .
$$

Finally, we have to consider spatial reflections at the $x_{1}$-axis (see Fig. 1) which act as

$$
R \vec{e}(x)=\vec{e}(R x), \quad R x=\left(x_{1},-x_{2}, t\right)=(r \cos \chi,-r \sin \chi, t),
$$

as well as time reversal, which changes the direction of a spin, and is represented by

$$
{ }^{T} \vec{e}(x)=-\vec{e}(T x), \quad T x=\left(x_{1}, x_{2},-t\right)=(r \cos \chi, r \sin \chi,-t) .
$$

The effective action, Eq. (2.1), respects all these symmetries which (except for the accidental Poincaré invariance) are inherited from the underlying Hubbard or $t-J$ models. 


\subsection{Skyrmion Solutions}

Skyrmions are topologically non-trivial classical solutions of the magnon effective field theory. Their integer winding number

$$
n[\vec{e}]=\frac{1}{8 \pi} \int d^{2} x \varepsilon_{i j} \vec{e} \cdot\left[\partial_{i} \vec{e} \times \partial_{j} \vec{e}\right] \in \Pi_{2}\left[S^{2}\right]=\mathbb{Z},
$$

takes values in the second homotopy group of the order parameter sphere $S^{2}$. The topological current

$$
j_{\mu}(x)=\frac{1}{8 \pi} \varepsilon_{\mu \nu \rho} \vec{e}(x) \cdot\left[\partial_{\nu} \vec{e}(x) \times \partial_{\rho} \vec{e}(x)\right],
$$

represents a conserved quantity, i.e. $\partial_{\mu} j_{\mu}(x)=0$. The time component of the topological current is related to the winding number by $n[\vec{e}]=\int d^{2} x j_{t}(x)$ and thus represents the integrated topological charge density. The transformation properties of the topological charge density with respect to the relevant symmetries are

$$
\begin{aligned}
U(1)_{s}: & { }^{I(\gamma)} j_{t}(x)=j_{t}(x), \\
D_{i}: & { }^{D} j_{t}(x)=j_{t}(x), \\
O: & { }^{O} j_{t}(x)=-j_{t}(O x), \\
O^{\prime}: & { }^{\prime} j_{t}(x)=-j_{t}(O x), \\
R: & { }^{R} j_{t}(x)=-j_{t}(R x), \\
T: & { }^{T} j_{t}(x)=-j_{t}(T x) .
\end{aligned}
$$

The winding number thus picks up a sign under the rotations $O$ and $O^{\prime}$, as well as under the reflection $R$ and time reversal $T$. Note that, in the case of the square lattice antiferromagnet, the displacements by one lattice spacing induce a sign change, while under 90 degrees rotations the winding number is invariant.

Static classical solutions minimize the energy functional given by

$$
E[\vec{e}]=\int d^{2} x \frac{\rho_{s}}{2} \partial_{i} \vec{e} \cdot \partial_{i} \vec{e} .
$$

Simple vector algebra,

$$
\begin{aligned}
0 & \leq \int d^{2} x\left(\partial_{i} \vec{e} \pm \varepsilon_{i j} \partial_{j} \vec{e} \times \vec{e}\right)^{2} \\
& =\int d^{2} x\left(2 \partial_{i} \vec{e} \cdot \partial_{i} \vec{e} \pm 2 \varepsilon_{i j} \vec{e} \cdot\left(\partial_{i} \vec{e} \times \partial_{j} \vec{e}\right)\right)=\frac{4}{\rho_{s}} E[\vec{e}] \pm 16 \pi n[\vec{e}]
\end{aligned}
$$

leads to the Schwarz inequality

$$
E[\vec{e}] \geq 4 \pi \rho_{s}|n[\vec{e}]|
$$


One distinguishes between Skyrmions that minimize the energy in the topological sector with winding number $n[\vec{e}]=1$, and anti-Skyrmions where $n[\vec{e}]=-1$. Classically both configurations have a rest energy of $\mathcal{M} c^{2}=4 \pi \rho_{s}$. According to Eq. (2.13), (anti-)Skyrmions satisfy the inequality Eq. (2.14) as an equality, provided that they obey the (anti-)self-duality equation

$$
\partial_{i} \vec{e}+\sigma \varepsilon_{i j} \partial_{j} \vec{e} \times \vec{e}=0 .
$$

Note that the quantity $\sigma$ refers to either Skyrmions $(\sigma=1)$ or anti-Skyrmions $(\sigma=-1)$. A particular (anti-)Skyrmion configuration, in polar coordinates $\left(x_{1}, x_{2}\right)=$ $r(\cos \chi, \sin \chi)$, is

$$
\vec{e}_{\sigma, n, \rho}(r, \chi)=\left(\frac{2 r^{n} \rho^{n}}{r^{2 n}+\rho^{2 n}} \cos (n \chi), \frac{2 r^{n} \rho^{n} \sigma}{r^{2 n}+\rho^{2 n}} \sin (n \chi), \frac{r^{2 n}-\rho^{2 n}}{r^{2 n}+\rho^{2 n}}\right) .
$$

In either case, the winding number is given by $n[\vec{e}]=\sigma n$, where $n \in \mathbb{N}_{>0}$. The (anti-)Skyrmion is centered at the origin and has size $\rho$.

It should be pointed out that the radial profile of the Skyrmion gets modified, when holes localize on a Skyrmion or anti-Skyrmion. In order to take this effect related to the details of the hole-Skyrmion interaction into account, we will allow for a general radial profile function $f(r) \in[-1,1]$ in our ansatz for the (anti-)Skyrmion configurations,

$$
\vec{e}_{\sigma, n, \rho}(r, \chi)=\left(\sqrt{1-f^{2}(r)} \cos (n \chi), \sigma \sqrt{1-f^{2}(r)} \sin (n \chi), f(r)\right)
$$

as we discuss in Sec. 4. The behavior of the profile function $f(r)$ at the origin and at infinity is the same as for the standard radial profile, i.e., $f(0)=-1$ and $f(\infty)=1$.

The Skyrmion configurations defined in Eq. (2.16) are characterized by a number of zero-modes. A shift to an arbitrary position $x$, spatial rotations

$$
O(\beta) x=(r \cos (\chi+\beta), r \sin (\chi+\beta), t),
$$

(where $x=(r \cos \chi, r \sin \chi, t)$ ) by an arbitrary angle $\beta$, or a $U(1)_{s}$ spin-rotation by an arbitrary angle $\gamma$, do not alter the Skyrmion's energy. As for the square lattice, also in the present case of the honeycomb lattice, spatial rotations and $U(1)_{s}$ spin rotations acting on a Skyrmion configuration are related by

$$
\begin{aligned}
{ }^{O(\beta)} \vec{e}_{\sigma, n, \rho}(r, \chi) & =\left(\frac{2 r^{n} \rho^{n}}{r^{2 n}+\rho^{2 n}} \cos (n(\chi+\beta)), \frac{2 r^{n} \rho^{n} \sigma}{r^{2 n}+\rho^{2 n}} \sin (n(\chi+\beta)), \frac{r^{2 n}-\rho^{2 n}}{r^{2 n}+\rho^{2 n}}\right), \\
{ }^{I(\sigma \gamma)} \vec{e}_{\sigma, n, \rho}(r, \chi) & =\left(\frac{2 r^{n} \rho^{n}}{r^{2 n}+\rho^{2 n}} \cos (n \chi+\gamma), \frac{2 r^{n} \rho^{n} \sigma}{r^{2 n}+\rho^{2 n}} \sin (n \chi+\gamma), \frac{r^{2 n}-\rho^{2 n}}{r^{2 n}+\rho^{2 n}}\right),
\end{aligned}
$$

such that

$$
{ }^{I(\sigma \gamma)} \vec{e}_{\sigma, n, \rho}(r, \chi)=O(\gamma / n) \vec{e}_{\sigma, n, \rho}(r, \chi) .
$$


Yet another zero-mode concerns dilations: under changes of the scale parameter $\rho$, the Skyrmion energy is not altered. We can create a family of Skyrmion configurations from the original Skyrmion defined in Eq. (2.16) by applying a spin rotation by an angle $\sigma \gamma$ and then performing a shift by a distance vector $x$,

$$
\vec{e}_{\sigma, n, \rho, x, \gamma}(r, \chi)=D(x)\left[{ }^{I(\sigma \gamma)} \vec{e}_{\sigma, n, \rho}(r, \chi)\right] .
$$

The transformation properties of these more general configurations under the various unbroken symmetry transformations are

$$
\begin{aligned}
U(1)_{s}: & I\left(\sigma \gamma_{0}\right) \vec{e}_{\sigma, n, \rho, x, \gamma}(r, \chi) & =\vec{e}_{\sigma, n, \rho, x, \gamma+\gamma_{0}}(r, \chi), \\
D: & D\left(x_{0}\right) \vec{e}_{\sigma, n, \rho, x, \gamma}(r, \chi) & =\vec{e}_{\sigma, n, \rho, x+x_{0}, \gamma}(r, \chi), \\
O(\beta): & { }^{(\beta)} \vec{e}_{\sigma, n, \rho, x, \gamma}(r, \chi) & =\vec{e}_{\sigma, n, \rho, O(\beta) x, \gamma+n \beta}(r, \chi), \\
O^{\prime}: & { }^{\prime} \vec{e}_{\sigma, n, \rho, x, \gamma}(r, \chi) & =\vec{e}_{-\sigma, n, \rho, O x, \gamma+\frac{\pi n}{3}}(r, \chi), \\
R: & R_{\vec{e}_{\sigma, n, \rho, x, \gamma}}(r, \chi) & =\vec{e}_{-\sigma, n, \rho, R x,-\gamma}(r, \chi) .
\end{aligned}
$$

It is worth pointing out that, unlike in particle physics where Skyrmions correspond to baryons, in antiferromagnets the Skyrmion number is not associated with the conserved fermion number in the underlying Hubbard model.

As we have discussed in detail for the square lattice case [12], apart from the integer winding number $n[\vec{e}]$, there is another topological invariant: the Hopf number $H[\vec{e}]$. While the former is defined at any instant of time, the latter describes the topology of the order parameter vector field $\vec{e}(x)$ as a function of both time and space. The Hopf number $H[\vec{e}] \in \Pi_{3}\left[S^{2}\right]=\mathbb{Z}$ takes integer values and is related to the third homotopy group of the sphere $S^{2}$. The transformation properties of the Hopf number under the various relevant symmetries are

$$
\begin{aligned}
U(1)_{s}: & \left.H{ }^{I(\gamma)} \vec{e}\right]=H[\vec{e}], \\
D_{i}: & \left.H^{D^{D}} \vec{e}\right]=H[\vec{e}], \\
O: & H\left[^{O} \vec{e}\right]=H[\vec{e}], \\
O^{\prime}: & H\left[^{O^{\prime}} \vec{e}\right]=H[\vec{e}], \\
R: & H\left[^{R} \vec{e}\right]=-H[\vec{e}], \\
T: & H\left[^{T} \vec{e}\right]=-H[\vec{e}] .
\end{aligned}
$$

The Euclidean path integral picks up an additional factor $\exp (i \Theta H[\vec{e}])$ when the Hopf term is included in the dynamics. The values of the anyon statistics angle $\Theta$ are restricted to 0 or $\pi$ for systems which are invariant under reflection or timereversal symmetry. Accordingly, the Skyrmions are quantized as bosons or fermions. If reflection and time-reversal do not represent symmetries of the systems, then $\Theta$ may take arbitrary values, and the spin of the Skyrmions need not be integer or halfinteger. While it is expected that the Hopf term is not present in doped cuprates 
[13, 14, 36 38], in the present study we include it, because we want to keep the discussion as general as possible.

In Ref. [12] we have performed the collective mode quantization of the Skyrmion in the undoped square lattice antiferromagnet, where the standard profile of the Skyrmion is relevant. In the present case of the honeycomb lattice, the analysis for the undoped system is exactly the same, and the interested reader may consult Section 2.4 of our earlier study for details. Here we just list some essential results of that analysis.

The quantum mechanical Hamiltonian describing Skyrmions on the honeycomb lattice takes the form

$$
H=\mathcal{M} c^{2}-\frac{1}{2 \mathcal{M}} \partial_{x_{i}}^{2}-\frac{1}{\sqrt{2 \mathcal{D}(\rho)}}\left(\partial_{\rho}^{2}+\frac{1}{\rho} \partial_{\rho}\right) \frac{1}{\sqrt{2 \mathcal{D}(\rho)}}-\frac{n^{2}}{2 \mathcal{D}(\rho) \rho^{2}}\left(\partial_{\gamma}+i n \frac{\Theta}{2 \pi}\right)^{2} .
$$

The explicit expressions for the rest energy, $\mathcal{M} c^{2}$, and the inertia of the Skyrmion with respect to dilations, $\mathcal{D}(\rho)$, can be found in Ref. [12]. It should be pointed out that for the standard profile, where the quantity $\mathcal{D}(\rho)$ is related to the moment of inertia $\mathcal{I}(\rho)$ of the Skyrmion by

$$
\mathcal{I}(\rho)=\frac{\mathcal{D}(\rho) \rho^{2}}{n^{2}},
$$

these two quantities are logarithmically divergent in the infrared for $n=1$. As described in Ref. [12], one may introduce an infrared cutoff to cure the problem.

The collective mode wave function referring to a Skyrmion or anti-Skyrmion characterized by its winding number $\sigma n$, momentum $p_{i}$, and spin $p_{\gamma}=\sigma m \in \mathbb{Z}$ amounts to

$$
\Psi_{p, \sigma, n, m}(x, \rho, \gamma)=\exp \left(i p_{i} x_{i}\right) \exp (i \sigma m \gamma) \psi(\rho) .
$$

Including the Hopf term in our analysis, the spin operator of the Skyrmion (i.e., the analog of isospin in particle physics) is given by

$$
I=\sigma\left(p_{\gamma}+n \frac{\Theta}{2 \pi}\right)=\sigma\left(-i \partial_{\gamma}+n \frac{\Theta}{2 \pi}\right) .
$$

The state $\Psi_{p, \sigma, n, m}(x, \rho, \gamma)$ thus corresponds to the "isospin"

$$
I \Psi_{p, \sigma, n, m}(x, \rho, \gamma)=\left(m+\sigma n \frac{\Theta}{2 \pi}\right) \Psi_{p, \sigma, n, m}(x, \rho, \gamma) .
$$

Note that for $\Theta=0$ the "isospin" takes integer values, whereas for $\Theta=\pi$ it is a half-integer for odd $n$.

Finally, according to Eq. (2.20), the angular momentum $J$ of a Skyrmion or antiSkyrmion turns out to be

$$
J=\sigma n I=n\left(p_{\gamma}+n \frac{\Theta}{2 \pi}\right)=n\left(-i \partial_{\gamma}+n \frac{\Theta}{2 \pi}\right)
$$


such that

$$
J \Psi_{p, \sigma, n, m}(x, \rho, \gamma)=n\left(\sigma m+n \frac{\Theta}{2 \pi}\right) \Psi_{p, \sigma, n, m}(x, \rho, \gamma) .
$$

For $\Theta=0$ the Skyrmion has integer angular momentum and hence represents a boson, while for $\Theta=\pi$ the angular momentum takes half-odd-integer values and the Skyrmion hence is a fermion. If the anyon statistics angle $\Theta$ is different from 0 or $\pi$, then we are dealing with anyons in $(2+1)$ dimensions, i.e., particles with any (neither integer nor half-integer) angular momentum.

\section{Effective Action for Hole-Doped Antiferromag- nets on the Honeycomb Lattice}

The effective Lagrangian for hole-doped antiferromagnets on the honeycomb lattice has been established in Ref. [2]. In this section we review some basic aspects of that systematic construction.

\subsection{Nonlinear Realization of the $S U(2)_{s}$ Symmetry}

One essential ingredient of the effective field theory analysis is the nonlinear realization of the spontaneously broken $S U(2)_{s}$ symmetry, which allows one to couple holes to the staggered magnetization order parameter [7]. The global $S U(2)_{s}$ symmetry then appears as a local $U(1)_{s}$ symmetry in the unbroken subgroup.

Let us parametrize the unit-vector order parameter field $\vec{e}_{\varphi}(x)$ as

$$
\vec{e}_{\varphi}(x)=(-\sin \varphi(x), \cos \varphi(x), 0) .
$$

The nonlinear realization of the $S U(2)_{s}$ symmetry is based on the matrix

$$
\begin{aligned}
u(x) & =\frac{1}{\sqrt{2\left(1+e_{3}(x)\right)}}\left(\begin{array}{cc}
1+e_{3}(x) & e_{1}(x)-i e_{2}(x) \\
-e_{1}(x)-i e_{2}(x) & 1+e_{3}(x)
\end{array}\right) \\
& =\left(\begin{array}{cc}
\cos \left(\frac{1}{2} \theta(x)\right) & \sin \left(\frac{1}{2} \theta(x)\right) \exp (-i \varphi(x)) \\
-\sin \left(\frac{1}{2} \theta(x)\right) \exp (i \varphi(x)) & \cos \left(\frac{1}{2} \theta(x)\right)
\end{array}\right) \\
& =\cos \left(\frac{1}{2} \theta(x)\right)+i \sin \left(\frac{1}{2} \theta(x)\right) \vec{e}_{\varphi}(x) \cdot \vec{\sigma} .
\end{aligned}
$$

Applying a global $S U(2)_{s}$ transformation $g$, the field $u(x)$ turns into

$$
u(x)^{\prime}=h(x) u(x) g^{\dagger}, \quad u_{11}(x)^{\prime} \geq 0 .
$$

By the above equation, the nonlinear symmetry transformation,

$$
h(x)=\exp \left(i \alpha(x) \sigma_{3}\right)=\left(\begin{array}{cc}
\exp (i \alpha(x)) & 0 \\
0 & \exp (-i \alpha(x))
\end{array}\right) \in U(1)_{s}
$$


is implicitly defined. We thus see that the global transformations $g \in S U(2)_{s}$, related to the spontaneously broken non-Abelian spin symmetry, manifest themselves as local transformations $h(x) \in U(1)_{s}$ in the unbroken Abelian subgroup. Note that the global subgroup transformations $I(\gamma)$, defined in Eq. (2.3), take the simple form $\alpha(x)=$ $-\gamma / 2$.

To construct the nonlinear realization of the $S U(2)_{s}$ symmetry, one proceeds with the diagonalizing matrix $u(x)$ defined in Eq. (3.2). The magnon field which then couples to the holes is the traceless anti-Hermitean field $v_{\mu}(x)$. It is obtained from the matrix $u(x)$ by

$$
v_{\mu}(x)=u(x) \partial_{\mu} u(x)^{\dagger} .
$$

Under the various symmetries, identified in the underlying microscopic $t$ - $J$ model, this effective field transforms as

$$
\begin{array}{rlrl}
S U(2)_{s}: & & v_{\mu}(x)^{\prime}=h(x)\left(v_{\mu}(x)+\partial_{\mu}\right) h(x)^{\dagger} \\
D_{i}: & { }^{D_{i}} v_{\mu}(x)=v_{\mu}(x) \\
O: & { }^{O} v_{1}(x)=\tau(O x)\left\{\frac{1}{2} v_{1}(O x)+\frac{\sqrt{3}}{2} v_{2}(O x)+\frac{1}{2} \partial_{1}+\frac{\sqrt{3}}{2} \partial_{2}\right\} \tau(O x)^{\dagger} \\
& { }^{O} v_{2}(x)=\tau(O x)\left\{-\frac{\sqrt{3}}{2} v_{1}(O x)+\frac{1}{2} v_{2}(O x)-\frac{\sqrt{3}}{2} \partial_{1}+\frac{1}{2} \partial_{2}\right\} \tau(O x)^{\dagger}, \\
& { }^{O} v_{t}(x)=\tau(O x)\left\{v_{t}(O x)+\partial_{t}\right\} \tau(O x)^{\dagger} \\
O^{\prime}: & { }^{O^{\prime}} v_{1}(x)=\frac{1}{2}\left(v_{1}(O x)^{*}+\sqrt{3} v_{2}(O x)^{*}\right) \\
& { }^{O^{\prime}} v_{2}(x)=\frac{1}{2}\left(-\sqrt{3} v_{1}(O x)^{*}+v_{2}(O x)^{*}\right) \\
& { }^{O^{\prime}} v_{t}(x)=v_{t}(O x)^{*}, \\
& { }^{R} v_{1}(x)=v_{1}(R x), \quad R v_{2}(x)=-v_{2}(R x) \\
& { }^{R} v_{t}(x)=v_{t}(R x), \\
& { }^{T} v_{i}(x)=\tau(T x)\left(v_{i}(T x)+\partial_{i}\right) \tau(T x)^{\dagger} \\
& { }^{T} v_{t}(x)=-\tau(T x)\left(v_{t}(T x)+\partial_{t}\right) \tau(T x)^{\dagger} .
\end{array}
$$

Note that the matrix $\tau(x)$ is defined in Eq. (3.20) of Ref. [2]. It is convenient to decompose $v_{\mu}(x)$ into an Abelian "gauge" field $v_{\mu}^{3}(x)$ and two "charged" vector fields $v_{\mu}^{ \pm}(x)$

$$
v_{\mu}(x)=i v_{\mu}^{a}(x) \sigma_{a}, \quad v_{\mu}^{ \pm}(x)=v_{\mu}^{1}(x) \mp i v_{\mu}^{2}(x) .
$$

Now for a Skyrmion $\vec{e}_{\sigma, n, \rho, 0, \gamma}(r, \chi)$ centered at $x=0$, according to Eq. (2.21), one 
ends up with

$$
\begin{aligned}
v_{1}^{3}(r, \chi) & =-\frac{\sigma n}{2 r}(1-f(r)) \sin \chi \\
v_{2}^{3}(r, \chi) & =\frac{\sigma n}{2 r}(1-f(r)) \cos \chi \\
v_{t}^{3}(r, \chi) & =\frac{\sigma}{2}(1-f(r)) \dot{\gamma} \\
v_{1}^{ \pm}(r, \chi) & =\frac{1}{2}\left(\mp \frac{i f^{\prime}(r)}{\sqrt{1-f^{2}(r)}} \cos \chi-\frac{\sigma n}{r} \sqrt{1-f^{2}(r)} \sin \chi\right) \exp (\mp i \sigma[n \chi+\gamma]), \\
v_{2}^{ \pm}(r, \chi) & =\frac{1}{2}\left(\mp \frac{i f^{\prime}(r)}{\sqrt{1-f^{2}(r)}} \sin \chi+\frac{\sigma n}{r} \sqrt{1-f^{2}(r)} \cos \chi\right) \exp (\mp i \sigma[n \chi+\gamma]), \\
v_{t}^{ \pm}(r, \chi) & =\frac{\sigma}{2} \sqrt{1-f^{2}(r)} \exp (\mp i \sigma(n \chi+\gamma)) \dot{\gamma} .
\end{aligned}
$$

Remember that the quantity $f(r)$, introduced in Eq. (2.17), describes a general radial profile function of the Skyrmion. It has to be pointed out that holes, when they get localized on a Skyrmion, do affect its radial profile. In the present study, we mainly focus on symmetry considerations, which are not affected by the actual form of the profile $f(r)$.

\subsection{Hole Fields and Transformation Properties}

In the effective field theory description, the holes are represented by Grassmann-valued fields $\psi_{ \pm}^{f}(x)[1]$. The index $f \in\{\alpha, \beta\}$ denotes the flavor of the momentum space pockets in which the holes reside. The subscript \pm , on the other hand, refers to the spin of the hole with respect to the direction of the local staggered magnetization. The transformation properties of the hole fields under the symmetries of the underlying honeycomb lattice antiferromagnet are as follows:

$$
\begin{array}{rlrl}
S U(2)_{s}: & \psi_{ \pm}^{f}(x)^{\prime} & =\exp ( \pm i \alpha(x)) \psi_{ \pm}^{f}(x), \\
U(1)_{Q}: & { }^{Q} \psi_{ \pm}^{f}(x) & =\exp (i \omega) \psi_{ \pm}^{f}(x), \\
D_{i}: & { }^{{ }}{ }_{i} \psi_{ \pm}^{f}(x) & =\exp \left(i k^{f} a_{i}\right) \psi_{ \pm}^{f}(x), \\
O: & { }^{O} \psi_{ \pm}^{\alpha}(x) & =\mp \exp \left( \pm i \frac{2 \pi}{3} \mp i \varphi(O x)\right) \psi_{\mp}^{\beta}(O x), \\
& { }^{O} \psi_{ \pm}^{\beta}(x)=\mp \exp \left(\mp i \frac{2 \pi}{3} \mp i \varphi(O x)\right) \psi_{\mp}^{\alpha}(O x), \\
O^{\prime}: & { }^{O^{\prime}} \psi_{ \pm}^{\alpha}(x)= \pm \exp \left( \pm i \frac{2 \pi}{3}\right) \psi_{\mp}^{\beta}(O x), \\
& { }^{\prime} \psi_{ \pm}^{\beta}(x)= \pm \exp \left(\mp i \frac{2 \pi}{3}\right) \psi_{\mp}^{\alpha}(O x), \\
R: & { }^{R} \psi_{ \pm}^{f}(x)=\psi_{ \pm}^{f^{\prime}}(R x) .
\end{array}
$$

While the $U(1)_{Q}$ symmetry refers to fermion number conservation, the quantities $k^{\alpha}=-k^{\beta}=\left(0, \frac{4 \pi}{3 \sqrt{3} a}\right)$ represent the lattice momenta of the pockets where doped holes 


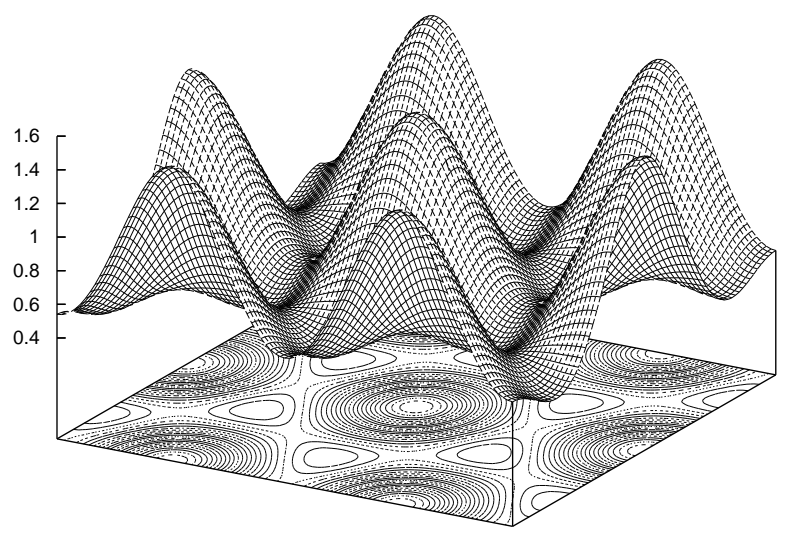

Figure 2: Dispersion relation $E(k) / t$ of a single hole doped into a honeycomb-lattice antiferromagnet, obtained from a simulation of the $t$ - $J$ model for $J / t=2$ [39].

of flavor $\alpha$ and $\beta$ reside. As illustrated in Fig. 2, holes indeed occupy spherically shaped hole pockets characterized by lattice momenta $\left( \pm \frac{2 \pi}{3 a}, \pm \frac{2 \pi}{3 \sqrt{3} a}\right)$ and $\left(0, \pm \frac{4 \pi}{3 \sqrt{3} a}\right)$.

\subsection{Effective Action}

Using the nonlinear realization of the magnon fields and the effective Grassmann field representation for the holes, it is straightforward to write down the leading and subleading terms of the effective action for a hole-doped antiferromagnet on the honeycomb lattice [2]. As in our earlier study of hole localization on a Skyrmion in a square-lattice antiferromagnet, here we also restrict ourselves to the leading terms in the effective action. Note however, that on the square lattice hole pockets are elliptically shaped. In Ref. [2] we considered the idealized case of circular hole pockets, in order to be able to perform large parts of the calculation analytically. Here, on the honeycomb lattice, hole pockets have a circular shape and we do not have to make any idealizations.

The action for hole-doped antiferromagnets on the honeycomb lattice then takes the form

$$
\begin{aligned}
S\left[\psi_{ \pm}^{f \dagger}, \psi_{ \pm}^{f}, \vec{e}\right] & =\int d^{2} x d t\left\{\frac{\rho_{s}}{2}\left(\partial_{i} \vec{e} \cdot \partial_{i} \vec{e}+\frac{1}{c^{2}} \partial_{t} \vec{e} \cdot \partial_{t} \vec{e}\right)+\sum_{\substack{f=\alpha, \beta \\
s=+,-}}\left[M \psi_{s}^{f \dagger} \psi_{s}^{f}\right.\right. \\
& \left.\left.+\psi_{s}^{f \dagger} D_{t} \psi_{s}^{f}+\frac{1}{2 M^{\prime}} D_{i} \psi_{s}^{f \dagger} D_{i} \psi_{s}^{f}+\Lambda \psi_{s}^{f \dagger}\left(i s v_{1}^{s}+\sigma_{f} v_{2}^{s}\right) \psi_{-s}^{f}\right]\right\}(3 .
\end{aligned}
$$


The quantities $M$ and $M^{\prime}$ are the rest mass and the kinetic mass of a hole, respectively. The low-energy effective coupling constant $\Lambda$ represents a hole-one-magnon coupling which, along with all other low-energy constants, takes real values. The sign $\sigma_{f}$ is + for $f=\alpha$ and - for $f=\beta$. The covariant derivatives are

$$
\begin{aligned}
& D_{t} \psi_{ \pm}^{f}(x)=\left[\partial_{t} \pm i v_{t}^{3}(x)-\mu\right] \psi_{ \pm}^{f}(x), \\
& D_{i} \psi_{ \pm}^{f}(x)=\left[\partial_{i} \pm i v_{i}^{3}(x)\right] \psi_{ \pm}^{f}(x) .
\end{aligned}
$$

Note that the chemical potential $\mu$ appears as an imaginary constant vector potential for fermion number $U(1)_{Q}$ in the covariant time derivative.

Remarkably, the $\Lambda$-term which contains just a single (uncontracted) spatial derivative is invariant under all the symmetries listed in Eq. (3.9). As it involves just one derivative, it represents the leading contribution to the low-energy dynamics of a lightly hole-doped honeycomb antiferromagnet. Note that for holed-doped square lattice antiferromagnets, such a term - the so-called Shraiman-Siggia term - is also present [1], whereas it is not allowed by symmetry reasons in the case of electron-doped square lattice antiferromagnets [10].

\subsection{Implications of the Shraiman-Siggia Term}

The Shraiman-Siggia term dominates the dynamics of the system at low energies. The explicit structure of this term depends both on the lattice geometry and on the localization of the hole pockets [1, 2]. In the case of the honeycomb lattice it takes the form $\Lambda \psi_{s}^{f \dagger}\left(i s v_{1}^{s}+\sigma_{f} v_{2}^{s}\right) \psi_{-s}^{f}$, while for the square lattice it is given by $\Lambda \psi_{s}^{f \dagger}\left(v_{1}^{s}+\right.$ $\left.\sigma_{f} v_{2}^{s}\right) \psi_{-s}^{f}$. As pointed out in Ref. [2], the $\Lambda$-term on the honeycomb lattice is invariant under an accidental global rotation symmetry by an arbitrary angle $\gamma$. For the square lattice, on the other hand, the $\Lambda$-term is only invariant under discrete rotations by 90 degrees.

This accidental rotation symmetry emerging on the honeycomb lattice has several important implications. First, the combination of magnon "gauge" fields in the $\Lambda$ term, according to Eqs. (3.8), implies

$$
\begin{aligned}
i v_{1}^{+}(x)+\sigma_{f} v_{2}^{+}(x)= & \frac{1}{2}\left[\frac{f^{\prime}(r)}{\sqrt{1-f^{2}(r)}}+\frac{\sigma \sigma_{f} n}{r} \sqrt{1-f(r)^{2}}\right] \\
& \times \exp \left(-i \sigma(n \chi+\gamma)-i \sigma_{f} \chi\right) \\
-i v_{1}^{-}(x)+\sigma_{f} v_{2}^{-}(x)= & \frac{1}{2}\left[\frac{f^{\prime}(r)}{\sqrt{1-f^{2}(r)}}+\frac{\sigma \sigma_{f} n}{r} \sqrt{1-f(r)^{2}}\right] \\
& \times \exp \left(i \sigma(n \chi+\gamma)+i \sigma_{f} \chi\right)
\end{aligned}
$$

and leads to the following interesting effect. The (anti-)Skyrmion standard profile, 
defined in Eq. (2.16), satisfies the differential equation

$$
\frac{f^{\prime}(r)}{\sqrt{1-f^{2}(r)}}-\frac{n}{r} \sqrt{1-f^{2}(r)}=0, \quad f(r)=\frac{r^{2 n}-\rho^{2 n}}{r^{2 n}+\rho^{2 n}} .
$$

Accordingly, for the standard profile, a Skyrmion can only localize a hole with flavor $\alpha$, while an anti-Skyrmion can only localize a hole of flavor $\beta$. For a general profile $f(r)$ on the honeycomb lattice, there is no such restriction. Note that on the square lattice, holes of flavor $\alpha$ and $\beta$ can localize both on Skyrmions and anti-Skyrmions for a general Skyrmion profile, including the standard one.

Another advantage of the honeycomb geometry is that the wave function of a single hole or of two holes localized on a (anti-)Skyrmion, factorizes into a radial and an angular part for an arbitrary profile function $f(r)$. As we will see in the next section, large parts of our calculations can thus be performed analytically. In the case of the square lattice, where an accidental rotation symmetry is absent, the factorization only occurs for the standard profile.

\section{Hole Localization on a Skyrmion}

This section deals with the application of the effective theory established in the previous section to the localization of holes on a Skyrmion. First, we consider the localization of a single hole, both on a static and on a rotating Skyrmion. Afterwards we investigate the localization of two holes of the same flavor on the same (anti-)Skyrmion, and analyze the symmetry properties of the emerging two-hole bound states.

\subsection{Single Hole Localized on a Static Skyrmion}

As it was shown in [12], the Skyrmion's moment of inertia $\mathcal{I}(\rho)$ is logarithmically divergent in the infrared for $n=1$ and for the standard profile. In this case, unless the divergence is regularized, the Skyrmion cannot rotate. Since in this study we focus on symmetry aspects and not on the details of the Skyrmion dynamics, we neglect the Skyrmion's translational and dilational motion, and fix the center of the Skyrmion at the origin $x=0$. We also fix the size of the Skyrmion to a constant radius $\rho$. If holes are incorporated, the energy of the Skyrmion-hole bound states takes a minimum for a particular value of $\rho$, as we will see later on.

If a single hole is localized on a Skyrmion, the corresponding wave function amounts to

$$
\Psi_{\sigma, n}^{f}(r, \chi)=\left(\begin{array}{c}
\Psi_{\sigma, n,+}^{f}(r, \chi) \\
\Psi_{\sigma, n,-}^{f}(r, \chi)
\end{array}\right)
$$


Since the rest energy $M$ of the holes just corresponds to a constant energy shift, this term can be neglected. The Hamiltonian resulting from the action of Eq.(3.10) thus takes the form

$$
\begin{aligned}
& H^{f}=\left(\begin{array}{cc}
H_{++}^{f} & H_{+-}^{f} \\
H_{-+}^{f} & H_{--}^{f}
\end{array}\right) \\
& H_{++}^{f}=-\frac{1}{2 M^{\prime}}\left[\partial_{i}+i v_{i}^{3}(x)\right]^{2}=-\frac{1}{2 M^{\prime}}\left[\partial_{r}^{2}+\frac{1}{r} \partial_{r}+\frac{1}{r^{2}}\left(\partial_{\chi}+i \frac{\sigma n}{2}(1-f(r))\right)^{2}\right] \text {, } \\
& H_{+-}^{f}=\Lambda\left(i v_{1}^{+}(x)+\sigma_{f} v_{2}^{+}(x)\right) \\
& =\frac{\Lambda}{2}\left[\frac{f^{\prime}(r)}{\sqrt{1-f^{2}(r)}}+\frac{\sigma \sigma_{f} n}{r} \sqrt{1-f^{2}(r)}\right] \exp \left(-i \sigma\left[\left(n+\sigma \sigma_{f}\right) \chi+\gamma\right]\right), \\
& H_{-+}^{f}=\Lambda\left(-i v_{1}^{-}(x)+\sigma_{f} v_{2}^{-}(x)\right) \\
& =\frac{\Lambda}{2}\left[\frac{f^{\prime}(r)}{\sqrt{1-f^{2}(r)}}+\frac{\sigma \sigma_{f} n}{r} \sqrt{1-f^{2}(r)}\right] \exp \left(i \sigma\left[\left(n+\sigma \sigma_{f}\right) \chi+\gamma\right]\right), \\
& H_{--}^{f}=-\frac{1}{2 M^{\prime}}\left[\partial_{i}-i v_{i}^{3}(x)\right]^{2}=-\frac{1}{2 M^{\prime}}\left[\partial_{r}^{2}+\frac{1}{r} \partial_{r}+\frac{1}{r^{2}}\left(\partial_{\chi}-i \frac{\sigma n}{2}(1-f(r))\right)^{2}\right] \text {. }
\end{aligned}
$$

With the explicit expressions for $v_{i}^{3}(x)$ and $v_{i}^{ \pm}(x)$ of the Skyrmion provided in Eq.(3.8), and making the ansatz

$$
\Psi_{\sigma, m_{+}^{f}, m_{-}^{f}}^{f}(r, \chi)=\left(\begin{array}{c}
\psi_{\sigma, m_{+}^{f}, m_{-}^{f},+}^{f}(r) \exp \left(i \sigma\left[m_{+}^{f} \chi-\frac{\gamma}{2}\right]\right) \\
\psi_{\sigma, m_{+}^{f}, m_{-}^{f},-}^{f}(r) \exp \left(i \sigma\left[m_{-}^{f} \chi+\frac{\gamma}{2}\right]\right)
\end{array}\right)
$$

with $m_{-}^{f}-m_{+}^{f}=n+\sigma \sigma_{f}$, one readily derives the radial Schrödinger equation

$$
H_{r}^{f} \psi_{\sigma, m_{+}^{f}, m_{-}^{f}}^{f}(r)=\left(\begin{array}{cc}
H_{r++}^{f} & H_{r+-}^{f} \\
H_{r-+}^{f} & H_{r--}^{f}
\end{array}\right)\left(\begin{array}{c}
\psi_{\sigma, m_{+}^{f}, m_{-}^{f},+}^{f}(r) \\
\psi_{\sigma, m_{+}^{f}, m_{-}^{f},-}^{f}(r)
\end{array}\right)=E_{m_{+}^{f}, m_{-}^{f}} \psi_{\sigma, m_{+}^{f}, m_{-}^{f}}^{f}(r),
$$

with

$$
\begin{aligned}
& H_{r++}^{f}=-\frac{1}{2 M^{\prime}}\left[\partial_{r}^{2}+\frac{1}{r} \partial_{r}-\frac{1}{r^{2}}\left(m_{+}^{f}+\frac{n}{2}(1-f(r))\right)^{2}\right], \\
& H_{r+-}^{f}=H_{r-+}^{f}=\frac{\Lambda}{2}\left[\frac{f^{\prime}(r)}{\sqrt{1-f^{2}(r)}}+\frac{\sigma \sigma_{f} n}{r} \sqrt{1-f^{2}(r)}\right], \\
& H_{r--}^{f}=-\frac{1}{2 M^{\prime}}\left[\partial_{r}^{2}+\frac{1}{r} \partial_{r}-\frac{1}{r^{2}}\left(m_{-}^{f}-\frac{n}{2}(1-f(r))\right)^{2}\right] .
\end{aligned}
$$

It should be pointed out that the emerging radial Schrödinger equation is not the same for Skyrmions and anti-Skyrmions, and neither is it identical for flavors $f=\alpha, \beta$. In the case of the square lattice, on the other hand, the resulting radial Schrödinger equation is the same for both flavors as well as for Skyrmions and anti-Skyrmions 
[12]. Interestingly, if $n$ is odd and if $m_{-}^{f}=-m_{+}^{f}=\left(n+\sigma \sigma_{f}\right) / 2$, the two equations decouple, and the equation that describes a localized hole amounts to

$$
\begin{aligned}
& \left\{-\frac{1}{2 M^{\prime}}\left(\partial_{r}^{2}+\frac{1}{r} \partial_{r}-\frac{1}{r^{2}}\left(\frac{n+\sigma \sigma_{f}}{2}-\frac{n}{2}(1-f(r))\right)^{2}\right)-\right. \\
& \left.\frac{\Lambda}{2}\left(\frac{f^{\prime}(r)}{\sqrt{1-f^{2}(r)}}+\frac{\sigma \sigma_{f} n}{r} \sqrt{1-f^{2}(r)}\right)\right\} \psi^{f}(r)=E \psi^{f}(r),
\end{aligned}
$$

where $\psi^{f}(r)$ corresponds to the linear combination

$$
\psi^{f}(r)=\frac{1}{\sqrt{2}}\left(\psi_{\sigma, m_{+}^{f}, m_{-}^{f},+}^{f}(r)-\psi_{\sigma, m_{+}^{f}, m_{-}^{f},-}^{f}(r)\right) .
$$

In the present study, we will be most interested in (anti-)Skyrmions with winding number $\sigma n= \pm 1$. For a Skyrmion or an anti-Skyrmion with $n=1$, the two equations decouple, but are still different for different flavors. For the Skyrmion (with $n=1$ and $\sigma=1$ ) the radial Schrödinger equation amounts to

$$
\begin{gathered}
{\left[-\frac{1}{2 M^{\prime}}\left(\partial_{r}^{2}+\frac{1}{r} \partial_{r}\right)+V^{f}(r)\right] \psi^{f}(r)=E \psi^{f}(r),} \\
V^{\alpha}(r)=\frac{1}{8 M^{\prime} r^{2}}(1+f(r))^{2}-\frac{\Lambda}{2}\left(\frac{f^{\prime}(r)}{\sqrt{1-f^{2}(r)}}+\frac{1}{r} \sqrt{1-f^{2}(r)}\right), \\
V^{\beta}(r)=\frac{1}{8 M^{\prime} r^{2}}(1-f(r))^{2}-\frac{\Lambda}{2}\left(\frac{f^{\prime}(r)}{\sqrt{1-f^{2}(r)}}-\frac{1}{r} \sqrt{1-f^{2}(r)}\right) .
\end{gathered}
$$

For the standard radial profile of the Skyrmion, given by $f(r)=\left(r^{2 n}-\rho^{2 n}\right) /\left(r^{2 n}+\rho^{2 n}\right)$, only $\alpha$-holes can be localized on the Skyrmion, since $\beta$-holes have a repulsive potential. Vice versa, $\alpha$-holes can not be localized on the anti-Skyrmion, but $\beta$-holes can be attracted by an anti-Skyrmion.

Although the main focus of the present study is a careful symmetry analysis, we still want to get a rough idea on the energy scales involved. Let us consider the standard Skyrmion profile and the situation where only one hole is localized. Here an attractive potential only emerges for an $\alpha$-hole localized on a Skyrmion (or a $\beta$-hole localized on an anti-Skyrmion), while the potential is repulsive in the other channel. Both the attractive and the repulsive potential are illustrated in Fig. 3. For $n=1$, the radial Schrödinger equation reduces to

$$
\left[-\frac{1}{2 M^{\prime}}\left(\partial_{r}^{2}+\frac{1}{r} \partial_{r}\right)+V^{\alpha}(r)\right] \psi^{\alpha}(r)=E \psi^{\alpha}(r),
$$

with the potential

$$
V^{\alpha}(r)=\frac{1}{2 M^{\prime}} \frac{r^{2}}{\left(r^{2}+\rho^{2}\right)^{2}}-2 \Lambda \frac{\rho}{r^{2}+\rho^{2}} .
$$




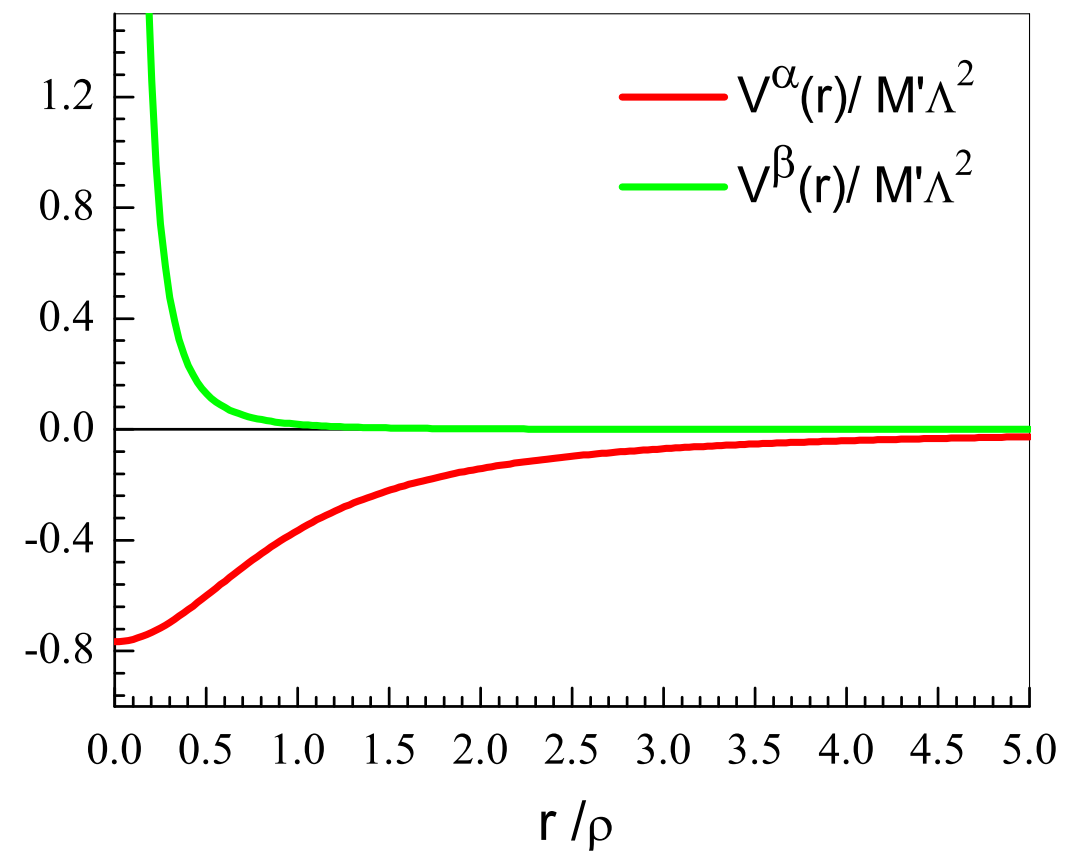

Figure 3: (Color online) The potentials $V^{\alpha}(r)$ and $V^{\beta}(r)$ related to the standard Skyrmion profile for $n=1$.

We now use the harmonic oscillator approximation, where at short distances the potential takes the form

$$
V_{\text {approx }}^{\alpha}(r)=-\frac{2 \Lambda}{\rho}+\frac{M^{\prime}}{2}\left(\frac{1}{M^{\prime 2} \rho^{4}}+\frac{4 \Lambda}{M^{\prime} \rho^{3}}\right) r^{2}+\mathcal{O}\left(r^{4}\right) .
$$

In this rather crude approximation, the ground state energy amounts to

$$
E_{0}=-\frac{2 \Lambda}{\rho}+\sqrt{\frac{1}{M^{\prime 2} \rho^{4}}+\frac{4 \Lambda}{M^{\prime} \rho^{3}}}=M^{\prime} \Lambda^{2} x\left(\sqrt{x^{2}+4 x}-2\right), \quad x=\frac{1}{M^{\prime} \Lambda \rho} .
$$

In terms of the parameter $x$, the minimization of the energy yields

$$
\begin{aligned}
& x=\frac{2}{\sqrt{3}}\left[\left(\frac{3 \sqrt{3}}{4}+\frac{\sqrt{11}}{4}\right)^{1 / 3}+\left(\frac{3 \sqrt{3}}{4}+\frac{\sqrt{11}}{4}\right)^{-1 / 3}\right]-2 \approx 0.383 \Rightarrow \\
& \rho \approx \frac{1}{0.383 M^{\prime} \Lambda} .
\end{aligned}
$$

The emerging bound state with the strongest binding energy is characterized by

$$
E_{0}=M^{\prime} \Lambda^{2} x\left(\sqrt{x^{2}+4 x}-2\right) \approx-0.270 M^{\prime} \Lambda^{2} .
$$

In Fig. 4 we have plotted the potential $V^{\alpha}(r)$ along with its harmonic approximation and the ground state energy $E_{0}$. According to this figure, the true energy of the ground state seems to be smaller than the harmonic approximation suggests. 


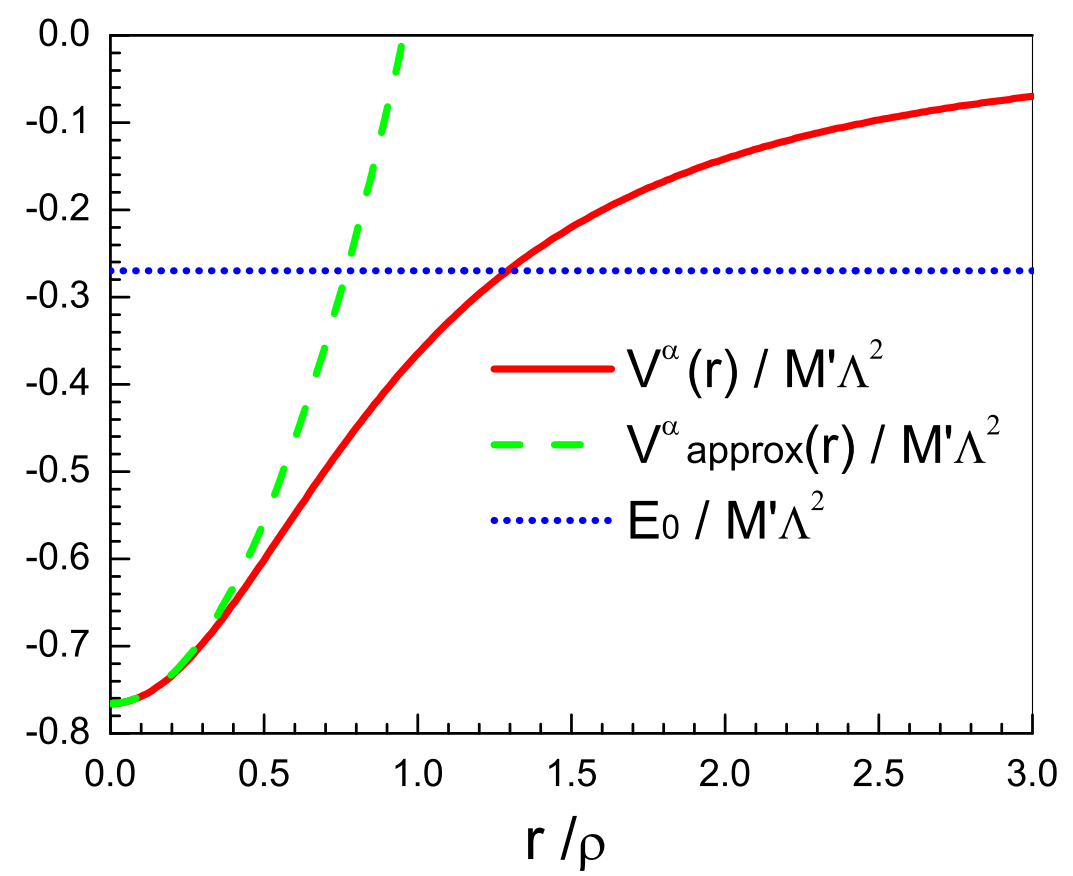

Figure 4: (Color online) The potential $V^{\alpha}(r)$ (solid line) along with its harmonic approximation (dashed line) and the resulting ground-state energy (dotted line).

\subsection{Single Hole Localized on a Rotating Skyrmion}

The Lagrange function for the rotational motion that involves $\gamma$ is given by

$$
L=\frac{\mathcal{D}(\rho) \rho^{2}}{2 n^{2}} \dot{\gamma}^{2}-n \frac{\Theta}{2 \pi} \dot{\gamma}+\int d^{2} x \sum_{\substack{f=\alpha, \beta \\ s=+,-}} s \psi_{s}^{f \dagger} v_{t}^{3} \psi_{s}^{f}
$$

The momentum canonically conjugate to $\gamma$, using Eq.(3.8), hence amounts to

$$
p_{\gamma}=\frac{\mathcal{D}(\rho) \rho^{2} \dot{\gamma}}{n^{2}}-n \frac{\Theta}{2 \pi}+\int d^{2} x \frac{\sigma}{2}(1-f(r)) \sum_{\substack{f=\alpha, \beta \\ s=+,-}} s \psi_{s}^{f \dagger} \psi_{s}^{f},
$$

leading to the Hamiltonian

$$
H^{\gamma}=\frac{1}{2 \mathcal{I}(\rho)}\left(-i \partial_{\gamma}-A_{\gamma}\right)^{2}
$$

where $A_{\gamma}$ is the Berry gauge field given by

$$
A_{\gamma}=\int d^{2} x \sum_{\substack{f=\alpha, \beta \\ s=+,-}} \Psi_{s}^{f \dagger} \frac{\sigma}{2}(1-f(r)) s \Psi_{s}^{f}-n \frac{\Theta}{2 \pi} .
$$


Combining the above results, one notices that the off-diagonal elements of the Hamiltonian (4.2) remain the same, whereas the diagonal elements involve additional contributions:

$$
\begin{aligned}
H^{f} & =\left(\begin{array}{cc}
H_{++}^{f} & H_{+-}^{f} \\
H_{-+}^{f} & H_{--}^{f}
\end{array}\right), \\
H_{++}^{f} & =-\frac{1}{2 M^{\prime}}\left[\partial_{r}^{2}+\frac{1}{r} \partial_{r}+\frac{1}{r^{2}}\left(\partial_{\chi}+i \frac{\sigma n}{2}(1-f(r))\right)^{2}\right] \\
& -\frac{n^{2}}{2 \mathcal{D}(\rho) \rho^{2}}\left(\partial_{\gamma}+i n \frac{\Theta}{2 \pi}-i \frac{\sigma}{2}(1-f(r))\right)^{2}, \\
H_{+-}^{f} & =\frac{\Lambda}{2}\left[\frac{f^{\prime}(r)}{\sqrt{1-f^{2}(r)}}+\frac{\sigma \sigma_{f} n}{r} \sqrt{1-f^{2}(r)}\right] \exp \left(-i \sigma\left[\left(n+\sigma \sigma_{f}\right) \chi+\gamma\right]\right), \\
H_{-+}^{f} & =\frac{\Lambda}{2}\left[\frac{f^{\prime}(r)}{\sqrt{1-f^{2}(r)}}+\frac{\sigma \sigma_{f} n}{r} \sqrt{1-f^{2}(r)}\right] \exp \left(i \sigma\left[\left(n+\sigma \sigma_{f}\right) \chi+\gamma\right]\right), \\
H_{--}^{f} & =-\frac{1}{2 M^{\prime}}\left[\partial_{r}^{2}+\frac{1}{r} \partial_{r}+\frac{1}{r^{2}}\left(\partial_{\chi}-i \frac{\sigma n}{2}(1-f(r))\right)^{2}\right] \\
& -\frac{n^{2}}{2 \mathcal{D}(\rho) \rho^{2}}\left(\partial_{\gamma}+i n \frac{\Theta}{2 \pi}+i \frac{\sigma}{2}(1-f(r))\right)^{2} .
\end{aligned}
$$

Our ansatz for the wave function is

$$
\Psi_{\sigma, m_{+}^{f}, m_{-}^{f}, m}^{f}(r, \chi, \gamma)=\left(\begin{array}{c}
\psi_{\sigma, m_{+}^{f}, m_{-}^{f}, m,+}^{f}(r) \exp \left(i \sigma m_{+}^{f} \chi\right) \exp \left(i \sigma\left(m-\frac{1}{2}\right) \gamma\right) \\
\psi_{\sigma, m_{+}^{f}, m_{-}^{f}, m,-}^{f}(r) \exp \left(i \sigma m_{-}^{f} \chi\right) \exp \left(i \sigma\left(m+\frac{1}{2}\right) \gamma\right)
\end{array}\right)
$$

with $m_{-}^{f}-m_{+}^{f}=n+\sigma \sigma_{f}$. Since the wave function must be $2 \pi$-periodic in the variable $\gamma$, the quantum number $m$ must now be one half of some odd integer. Note that in the case of the rotating Skyrmion without a hole, discussed in Subsection 2.2 (collective mode quantization), $m$ was an integer. The radial Schrödinger equation then takes the form

$$
\begin{aligned}
H_{r}^{f} \psi_{\sigma, m_{+}^{f}, m_{-}^{f}, m}^{f}(r) & =\left(\begin{array}{cc}
H_{r++}^{f} & H_{r+-}^{f} \\
H_{r-+}^{f} & H_{r--}^{f}
\end{array}\right)\left(\begin{array}{c}
\psi_{\sigma, m_{+}^{f}, m_{-}^{f}, m,+}^{f}(r) \\
\psi_{\sigma, m_{+}^{f}, m_{-}^{f}, m,-}^{f}(r)
\end{array}\right) \\
& =E_{\sigma, m_{+}^{f}, m_{-}^{f}, m} \psi_{\sigma, m_{+}^{f}, m_{-}^{f}, m}^{f}(r),
\end{aligned}
$$


where the four matrix elements of the radial Hamiltonian $H_{r}^{f}$ are

$$
\begin{aligned}
H_{r++}^{f} & =-\frac{1}{2 M^{\prime}}\left[\partial_{r}^{2}+\frac{1}{r} \partial_{r}-\frac{1}{r^{2}}\left(m_{+}^{f}+\frac{n}{2}(1-f(r))\right)^{2}\right] \\
& +\frac{n^{2}}{2 \mathcal{D}(\rho) \rho^{2}}\left(m-\frac{1}{2}+\sigma n \frac{\Theta}{2 \pi}-\frac{1}{2}(1-f(r))\right)^{2}, \\
H_{r+-}^{f} & =H_{r-+}^{f}=\frac{\Lambda}{2}\left[\frac{f^{\prime}(r)}{\sqrt{1-f^{2}(r)}}+\frac{\sigma \sigma_{f} n}{r} \sqrt{1-f^{2}(r)}\right], \\
H_{r--}^{f} & =-\frac{1}{2 M^{\prime}}\left[\partial_{r}^{2}+\frac{1}{r} \partial_{r}-\frac{1}{r^{2}}\left(m_{-}^{f}-\frac{n}{2}(1-f(r))\right)^{2}\right] \\
& +\frac{n^{2}}{2 \mathcal{D}(\rho) \rho^{2}}\left(m+\frac{1}{2}+\sigma n \frac{\Theta}{2 \pi}+\frac{1}{2}(1-f(r))\right)^{2} .
\end{aligned}
$$

\subsection{Single Hole Localized on a Rotating Skyrmion: Symme- try Properties}

Recall that the spin operator (which is related to an internal symmetry transformation and hence is analogous to isospin in particle physics)

$$
I=\left(\begin{array}{cc}
-i \sigma \partial_{\gamma}+\sigma n \frac{\Theta}{2 \pi}+\frac{1}{2} & 0 \\
0 & -i \sigma \partial_{\gamma}+\sigma n \frac{\Theta}{2 \pi}-\frac{1}{2}
\end{array}\right),
$$

commutes with the Hamiltonian: $\left[H^{f}, I\right]=0$. The wave function $\Psi_{\sigma, m_{+}^{f}, m_{-}^{f}, m}^{f}$ is then an eigenstate of $I$, i.e.

$$
I \Psi_{\sigma, m_{+}^{f}, m_{-}^{f}, m}^{f}(r, \chi, \gamma)=\left(m+\sigma n \frac{\Theta}{2 \pi}\right) \Psi_{\sigma, m_{+}^{f}, m_{-}^{f}, m}^{f}(r, \chi, \gamma)
$$

Because $m$ is half of an odd integer, the rotating Skyrmion with one hole localized on it carries half-integer spin (or "isospin"), provided that the anyon statistics parameter vanishes: $\Theta=0$.

Under the various discrete symmetries - the displacements $D_{1}$ and $D_{2}$, the rotation $O^{\prime}$, and the reflection $R$ - the wave function

$$
\Psi_{\sigma, n}^{f}(r, \chi, \gamma)=\left(\begin{array}{c}
\Psi_{\sigma, n,+}^{f}(r, \chi, \gamma) \\
\Psi_{\sigma, n,-}^{f}(r, \chi, \gamma)
\end{array}\right)
$$

describing a single hole localized on a rotating (anti-)Skyrmion with winding number 
$\sigma n$, transforms as

$$
\begin{aligned}
& { }^{D_{i}} \Psi_{\sigma, n}^{f}(r, \chi, \gamma)=\exp \left(i k^{f} a_{i}\right)\left(\begin{array}{c}
\Psi_{\sigma, n,+}^{f}(r, \chi, \gamma) \\
\Psi_{\sigma, n,-}^{f}(r, \chi, \gamma)
\end{array}\right) \\
& { }^{\prime} \Psi_{\sigma, n}^{f}(r, \chi, \gamma)=\left(\begin{array}{c}
\exp \left(-\sigma_{f} \frac{2 \pi i}{3}\right) \Psi_{\sigma, n,-}^{f}\left(r, \chi+\frac{\pi}{3}, \gamma-n \frac{\pi}{3}\right) \\
-\exp \left(\sigma_{f} \frac{2 \pi i}{3}\right) \Psi_{\sigma, n,+}^{f}\left(r, \chi+\frac{\pi}{3}, \gamma-n \frac{\pi}{3}\right)
\end{array}\right), \\
& { }^{R} \Psi_{\sigma, n}^{f}(r, \chi, \gamma)=\left(\begin{array}{c}
\Psi_{\sigma, n,+}^{f}(r,-\chi,-\gamma) \\
\Psi_{\sigma, n,-}^{f}(r,-\chi,-\gamma)
\end{array}\right)
\end{aligned}
$$

Accordingly, the energy eigenstates transform as

$$
\begin{aligned}
D_{i} \Psi_{\sigma, m_{+}^{f}, m_{-}^{f}, m}^{f}(r, \chi, \gamma) & =\exp \left(i k^{f} a_{i}\right) \Psi_{\sigma, m_{+}^{f}, m_{-}^{f}, m}^{f}(r, \chi, \gamma), \\
{ }^{\prime} \Psi_{\sigma, m_{+}^{\alpha}, m_{-}^{\alpha}, m}^{\alpha}(r, \chi, \gamma) & =\exp \left(i \sigma\left[m_{+}^{\alpha}+\frac{n}{2}-\sigma-m n\right] \frac{\pi}{3}\right) \Psi_{-\sigma,-m_{-}^{\alpha},-m_{+}^{\alpha},-m}^{\beta}(r, \chi, \gamma), \\
{ }^{\prime} \Psi_{\sigma, m_{+}, m_{-}^{\beta}, m}^{\beta}(r, \chi, \gamma) & =\exp \left(i \sigma\left[m_{+}^{\beta}+\frac{n}{2}+\sigma-m n\right] \frac{\pi}{3}\right) \Psi_{-\sigma,-m_{-}^{\beta},-m_{+}^{\beta},-m}^{\alpha}(r, \chi, \gamma), \\
{ }^{R} \Psi_{\sigma, m_{+}^{\alpha}, m_{-}^{\alpha}, m}^{\alpha}(r, \chi, \gamma) & =\Psi_{-\sigma, m_{+}^{\alpha}, m_{-}^{\alpha}, m}^{\beta}(r, \chi, \gamma), \\
{ }^{R} \Psi_{\sigma, m_{+}^{\beta}, m_{-}^{\beta}, m}^{\beta}(r, \chi, \gamma) & =\Psi_{-\sigma, m_{+}, m_{-}^{\beta}, m}^{\alpha}(r, \chi, \gamma) .
\end{aligned}
$$

Note that for $\Theta \neq 0$ or $\pi$, the Hopf term explicitly breaks the reflection symmetry $R$. Deriving these equations, we have used appropriate phase conventions for the radial wave functions. For the rotation symmetry $O^{\prime}$, we have used $\alpha^{\prime}=\beta, \beta^{\prime}=\alpha$, as well as

$$
\psi_{\sigma, m_{+}^{f}, m_{-}^{f}, m, \mp}^{f}(r)=\psi_{-\sigma,-m_{-}^{f},-m_{+}^{f},-m, \pm}^{f^{\prime}}(r),
$$

which results from the behavior of Eq.(4.24) by making the replacements $m_{+}^{f} \rightarrow m_{+}^{f^{\prime}}=$ $-m_{-}^{f}, m_{-}^{f} \rightarrow m_{-}^{f^{\prime}}=-m_{+}^{f}$, and $m \rightarrow m^{\prime}=-m$. For the reflection $R$ we have used

$$
\psi_{\sigma, m_{+}^{f}, m_{-}^{f}, m, \pm}^{f}(r)=\psi_{-\sigma, m_{+}^{f}, m_{-}^{f}, m, \pm}^{f^{\prime}}(r) .
$$

Note that after these replacements the constraint

$$
m_{-}^{f}-m_{+}^{f}=n+\sigma \sigma_{f}
$$

is still satisfied.

\subsection{Schrödinger Equation for a Hole Pair with the Same Fla- vor Localized on a Rotating Skyrmion}

As we discussed before, a Skyrmion with the standard radial profile can only localize $\alpha$-holes, while an anti-Skyrmion can localize $\beta$-holes. Here we consider a general radial profile and study an $\alpha \alpha$ pair localized on a rotating Skyrmion, or a $\beta \beta$ pair localized 
on a rotating anti-Skyrmion. It should be noted that the following symmetry analysis even applies to an $\alpha \alpha$ pair localized on an anti-Skyrmion or a $\beta \beta$ pair localized on a Skyrmion, but these configurations are not energetically favorable.

If bound states of two holes of the same flavor $f$ localize on a rotating Skyrmion, due to the Pauli principle, the holes cannot occupy the same state. Let us therefore distinguish the holes by an unphysical label 1 or 2 . If the wave function is antisymmetric under the exchange of these labels, the Pauli principle is satisfied. In this subsection we consider two holes of the same flavor which is most relevant at low energies and relegate the localization of two holes with different flavor to the Appendix.

The Hamiltonian describing two holes of the same flavor $f$ then reads

$$
H=H^{1}+H^{2}+H^{\gamma},
$$

where

$$
\begin{aligned}
H^{1} & =\left(\begin{array}{cccc}
H_{++}^{f} & 0 & H_{+-}^{f} & 0 \\
0 & H_{++}^{f} & 0 & H_{+-}^{f} \\
H_{-+}^{f} & 0 & H_{--}^{f} & 0 \\
0 & H_{-+}^{f} & 0 & H_{--}^{f}
\end{array}\right), \quad H^{2}=\left(\begin{array}{cccc}
H_{++}^{f} & H_{+-}^{f} & 0 & 0 \\
H_{-+}^{f} & H_{--}^{f} & 0 & 0 \\
0 & 0 & H_{++}^{f} & H_{+-}^{f} \\
0 & 0 & H_{-+}^{f} & H_{--}^{f}
\end{array}\right), \\
H^{\gamma} & =\left(\begin{array}{cccc}
H_{++++}^{\gamma} & 0 & 0 & 0 \\
0 & H_{+-+-}^{\gamma} & 0 & 0 \\
0 & 0 & H_{-+-+}^{\gamma} & 0 \\
0 & 0 & 0 & H_{----}^{\gamma}
\end{array}\right),
\end{aligned}
$$

with

$$
\begin{aligned}
& H_{++}^{\alpha}=-\frac{1}{2 M^{\prime}}\left(\partial_{i}+i v_{i}^{3}(x)\right)^{2}, \quad H_{+-}^{\alpha}=\Lambda\left(i v_{1}^{+}(x)+v_{2}^{+}(x)\right), \\
& H_{--}^{\alpha}=-\frac{1}{2 M^{\prime}}\left(\partial_{i}-i v_{i}^{3}(x)\right)^{2}, \quad H_{-+}^{\alpha}=\Lambda\left(-i v_{1}^{-}(x)+v_{2}^{-}(x)\right) \\
& H_{++}^{\beta}=-\frac{1}{2 M^{\prime}}\left(\partial_{i}+i v_{i}^{3}(x)\right)^{2}, \quad H_{+-}^{\beta}=\Lambda\left(i v_{1}^{+}(x)-v_{2}^{+}(x)\right), \\
& H_{--}^{\beta}=-\frac{1}{2 M^{\prime}}\left(\partial_{i}-i v_{i}^{3}(x)\right)^{2}, \quad H_{-+}^{\beta}=\Lambda\left(-i v_{1}^{-}(x)-v_{2}^{-}(x)\right), \\
& H_{++++}^{\gamma}=-\frac{n^{2}}{2 \mathcal{D}(\rho) \rho^{2}}\left(\partial_{\gamma}+i n \frac{\Theta}{2 \pi}-i \frac{\sigma}{2}\left(1-f\left(r_{\alpha}\right)\right)-i \frac{\sigma}{2}\left(1-f\left(r_{\beta}\right)\right)\right)^{2}, \\
& H_{+-+-}^{\gamma}=-\frac{n^{2}}{2 \mathcal{D}(\rho) \rho^{2}}\left(\partial_{\gamma}+i n \frac{\Theta}{2 \pi}-i \frac{\sigma}{2}\left(1-f\left(r_{\alpha}\right)\right)+i \frac{\sigma}{2}\left(1-f\left(r_{\beta}\right)\right)\right)^{2}, \\
& H_{-+-+}^{\gamma}=-\frac{n^{2}}{2 \mathcal{D}(\rho) \rho^{2}}\left(\partial_{\gamma}+i n \frac{\Theta}{2 \pi}+i \frac{\sigma}{2}\left(1-f\left(r_{\alpha}\right)\right)-i \frac{\sigma}{2}\left(1-f\left(r_{\beta}\right)\right)\right)^{2}, \\
& H_{----}^{\gamma}=-\frac{n^{2}}{2 \mathcal{D}(\rho) \rho^{2}}\left(\partial_{\gamma}+i n \frac{\Theta}{2 \pi}+i \frac{\sigma}{2}\left(1-f\left(r_{\alpha}\right)\right)+i \frac{\sigma}{2}\left(1-f\left(r_{\beta}\right)\right)\right)^{2} .(4.3
\end{aligned}
$$


We first ignore the Pauli principle, and relegate the antisymmetrization of the wave function in the labels 1 and 2 to the next section. This leads us to the following ansatz for an energy eigenstate of two holes, which we have distinguished by their labels,

$$
\begin{aligned}
& \Psi_{\sigma, m_{+}^{1}, m_{-}^{1}, m_{+}^{2}, m_{-}^{2}, m}^{f f}\left(r_{1}, \chi_{1}, r_{2}, \chi_{2}, \gamma\right)= \\
& \left(\begin{array}{c}
\psi_{\sigma, m_{+}^{+}, m_{-}^{1}, m_{+}^{2}, m_{-}^{2}, m,++}^{f f}\left(r_{1}, r_{2}\right) \exp \left(i \sigma\left[m_{+}^{1} \chi_{1}+m_{+}^{2} \chi_{2}\right]\right) \exp (i \sigma(m-1) \gamma) \\
\psi_{\sigma, m_{+}^{1}, m_{-}^{1}, m_{+}^{2}, m_{-}^{2}, m,+-}^{f f}\left(r_{1}, r_{2}\right) \exp \left(i \sigma\left[m_{+}^{1} \chi_{1}+m_{-}^{2} \chi_{2}\right]\right) \exp (i \sigma m \gamma) \\
\psi_{\sigma, m_{+}^{1}, m_{-}^{1}, m_{+}^{2}, m_{-}^{2}, m,-+}^{f f}\left(r_{1}, r_{2}\right) \exp \left(i \sigma\left[m_{-}^{1} \chi_{1}+m_{+}^{2} \chi_{2}\right]\right) \exp (i \sigma m \gamma) \\
\psi_{\sigma, m_{+}^{1}, m_{-}^{1}, m_{+}^{2}, m_{-}^{2}, m,--}^{f f}\left(r_{1}, r_{2}\right) \exp \left(i \sigma\left[m_{-}^{1} \chi_{1}+m_{-}^{2} \chi_{2}\right]\right) \exp (i \sigma(m+1) \gamma)
\end{array}\right) .
\end{aligned}
$$

This ansatz solves the Schrödinger equation, provided that $m_{-}^{i f}-m_{+}^{i f}=n+\sigma \sigma_{f}$, $i=1,2$. In this case, $m$ takes integer values and the radial Schrödinger equation amounts to

$$
H_{r} \psi_{\sigma, m_{+}^{1}, m_{-}^{1}, m_{+}^{2}, m_{-}^{2}, m}^{f f}\left(r_{1}, r_{2}\right)=E_{\sigma, m_{+}^{1}, m_{-}^{1}, m_{+}^{2}, m_{-}^{2}, m} \psi_{\sigma, m_{+}^{1}, m_{-}^{1}, m_{+}^{2}, m_{-}^{2}, m}^{f f}\left(r_{1}, r_{2}\right)
$$

with

$$
\psi_{\sigma, m_{+}^{1}, m_{-}^{1}, m_{+}^{2}, m_{-}^{2}, m}^{f f}\left(r_{1}, r_{2}\right)=\left(\begin{array}{c}
\psi_{\sigma, m_{+}^{1}, m_{-}^{1}, m_{+}^{2}, m_{-}^{2}, m,++}^{f f}\left(r_{1}, r_{2}\right) \\
\psi_{\sigma, m_{+}^{1}, m_{-}^{1}, m_{+}^{2}, m_{-}^{2}, m,+-}^{f f}\left(r_{1}, r_{2}\right) \\
\psi_{\sigma, m_{+}^{1}, m_{-}^{1}, m_{+}^{2}, m_{-}^{2}, m,-+}^{f f}\left(r_{1}, r_{2}\right) \\
\psi_{\sigma, m_{+}^{1}, m_{-}^{1}, m_{+}^{2}, m_{-}^{2}, m,--}^{f f}\left(r_{1}, r_{2}\right)
\end{array}\right) .
$$

The radial Hamiltonian is

$$
H_{r}=H_{r}^{1}+H_{r}^{2}+H_{r}^{\gamma}
$$

with

$$
\begin{aligned}
H_{r}^{1}= & \left(\begin{array}{cccc}
H_{r++}^{1} & 0 & H_{r+-}^{1} & 0 \\
0 & H_{r++}^{1} & 0 & H_{r+-}^{1} \\
H_{r-+}^{1} & 0 & H_{r--}^{1} & 0 \\
0 & H_{r-+}^{1} & 0 & H_{r--}^{1}
\end{array}\right), \\
H_{r}^{2}= & \left(\begin{array}{cccc}
H_{r++}^{2} & H_{r+-}^{2} & 0 & 0 \\
H_{r-+}^{2} & H_{r--}^{2} & 0 & 0 \\
0 & 0 & H_{r++}^{2} & H_{r+-}^{2} \\
0 & 0 & H_{r-+}^{2} & H_{r--}^{2}
\end{array}\right), \\
H_{r}^{\gamma}= & \left(\begin{array}{cccc}
H_{r++++}^{\gamma} & 0 & 0 & 0 \\
0 & H_{r+-+-}^{\gamma} & 0 & 0 \\
0 & 0 & H_{r-+-+}^{\gamma} & 0 \\
0 & 0 & 0 & H_{r----}^{\gamma}
\end{array}\right) .
\end{aligned}
$$


While the matrix elements related to the fermionic part of the radial Hamiltonian are

$$
\begin{aligned}
& H_{r++}^{i}=-\frac{1}{2 M^{\prime}}\left[\partial_{r_{i}}^{2}+\frac{1}{r_{i}} \partial_{r_{i}}-\frac{1}{r_{i}^{2}}\left(m_{+}^{i f}+\frac{n \rho^{2 n}}{r_{i}^{2 n}+\rho^{2 n}}\right)^{2}\right], \\
& H_{r+-}^{i}=H_{r-+}^{i}=2 \Lambda \frac{n r_{i}^{n-1} \rho^{n}}{r_{i}^{2 n}+\rho^{2 n}}, \\
& H_{r--}^{i}=-\frac{1}{2 M^{\prime}}\left[\partial_{r_{i}}^{2}+\frac{1}{r_{i}} \partial_{r_{i}}-\frac{1}{r_{i}^{2}}\left(m_{-}^{i f}-\frac{n \rho^{2 n}}{r_{i}^{2 n}+\rho^{2 n}}\right)^{2}\right],
\end{aligned}
$$

the contributions referring to the rotational Skyrmion read

$$
\begin{aligned}
& H_{r++++}^{\gamma}=\frac{n^{2}}{2 \mathcal{D}(\rho) \rho^{2}}\left(m-1+\sigma n \frac{\Theta}{2 \pi}-\frac{\rho^{2 n}}{r_{1}^{2 n}+\rho^{2 n}}-\frac{\rho^{2 n}}{r_{2}^{2 n}+\rho^{2 n}}\right)^{2}, \\
& H_{r+-+-}^{\gamma}=\frac{n^{2}}{2 \mathcal{D}(\rho) \rho^{2}}\left(m+\sigma n \frac{\Theta}{2 \pi}-\frac{\rho^{2 n}}{r_{1}^{2 n}+\rho^{2 n}}+\frac{\rho^{2 n}}{r_{2}^{2 n}+\rho^{2 n}}\right)^{2}, \\
& H_{r-+-+}^{\gamma}=\frac{n^{2}}{2 \mathcal{D}(\rho) \rho^{2}}\left(m+\sigma n \frac{\Theta}{2 \pi}+\frac{\rho^{2 n}}{r_{1}^{2 n}+\rho^{2 n}}-\frac{\rho^{2 n}}{r_{2}^{2 n}+\rho^{2 n}}\right)^{2}, \\
& H_{r----}^{\gamma}=\frac{n^{2}}{2 \mathcal{D}(\rho) \rho^{2}}\left(m+1+\sigma n \frac{\Theta}{2 \pi}+\frac{\rho^{2 n}}{r_{1}^{2 n}+\rho^{2 n}}+\frac{\rho^{2 n}}{r_{2}^{2 n}+\rho^{2 n}}\right)^{2} .
\end{aligned}
$$

\subsection{Hole Pair of the Same Flavor Localized on a Skyrmion: Symmetry Properties}

The spin operator $I$, which commutes with the two-hole Hamiltonian $H$, amounts to

$$
I=\left(\begin{array}{cccc}
-i \sigma \partial_{\gamma}+\sigma n \frac{\Theta}{2 \pi}+1 & 0 & 0 & 0 \\
0 & -i \sigma \partial_{\gamma}+\sigma n \frac{\Theta}{2 \pi} & 0 & 0 \\
0 & 0 & -i \sigma \partial_{\gamma}+\sigma n \frac{\Theta}{2 \pi} & 0 \\
0 & 0 & 0 & -i \sigma \partial_{\gamma}+\sigma n \frac{\Theta}{2 \pi}-1
\end{array}\right)
$$

such that

$$
\begin{aligned}
& I \Psi_{\sigma, m_{+}^{1}, m_{-}^{1}, m_{+}^{2}, m_{-}^{2}, m}^{f f}\left(r_{1}, \chi_{1}, r_{2}, \chi_{2}, \gamma\right)= \\
& \left(m+\sigma n \frac{\Theta}{2 \pi}\right) \Psi_{\sigma, m_{+}^{1}, m_{-}^{1}, m_{+}^{2}, m_{-}^{2}, m}^{f f}\left(r_{1}, \chi_{1}, r_{2}, \chi_{2}, \gamma\right) .
\end{aligned}
$$

Since $m$ takes integer values, at least for $\Theta=0$, the state containing two holes of the same flavor localized on a Skyrmion has integer spin as well. 
Under the symmetries $D_{i}, O^{\prime}$, and $R$ the two-hole wave function

$$
\Psi_{\sigma, n}^{f f}\left(r_{1}, \chi_{1}, r_{2}, \chi_{2}, \gamma\right)=\left(\begin{array}{c}
\Psi_{\sigma, n,++}^{f f}\left(r_{1}, \chi_{1}, r_{2}, \chi_{2}, \gamma\right) \\
\Psi_{\sigma, n,+-}^{f f}\left(r_{1}, \chi_{1}, r_{2}, \chi_{2}, \gamma\right) \\
\Psi_{\sigma, n,-+}^{f f}\left(r_{1}, \chi_{1}, r_{2}, \chi_{2}, \gamma\right) \\
\Psi_{\sigma, n,--}^{f f}\left(r_{1}, \chi_{1}, r_{2}, \chi_{2}, \gamma\right)
\end{array}\right)
$$

transforms as

$$
\begin{aligned}
{ }^{D_{i}} \Psi_{\sigma, n}^{f f}\left(r_{1}, \chi_{1}, r_{2}, \chi_{2}, \gamma\right)= & \exp \left(2 i k^{f} a_{i}\right)\left(\begin{array}{c}
\Psi_{\sigma, n,++}^{f f}\left(r_{1}, \chi_{1}, r_{2}, \chi_{2}, \gamma\right) \\
\Psi_{\sigma, n,+-}^{f f}\left(r_{1}, \chi_{1}, r_{2}, \chi_{2}, \gamma\right) \\
\Psi_{\sigma, n,-+}^{f f}\left(r_{1}, \chi_{1}, r_{2}, \chi_{2}, \gamma\right) \\
\Psi_{\sigma, n,--}^{f f}\left(r_{1}, \chi_{1}, r_{2}, \chi_{2}, \gamma\right)
\end{array}\right), \\
{ }^{\prime} \Psi_{\sigma, n}^{f f}\left(r_{1}, \chi_{1}, r_{2}, \chi_{2}, \gamma\right)= & \left(\begin{array}{c}
\exp \left(-\sigma_{f} \frac{4 \pi i}{3}\right) \Psi_{\sigma, n,--}^{f f}\left(r_{1}, \chi_{1}+\frac{\pi}{3}, r_{2}, \chi_{2}+\frac{\pi}{3}, \gamma-n \frac{\pi}{3}\right) \\
-\Psi_{\sigma, n,-+}^{f f}\left(r_{1}, \chi_{1}+\frac{\pi}{3}, r_{2}, \chi_{2}+\frac{\pi}{3}, \gamma-n \frac{\pi}{3}\right) \\
-\Psi_{\sigma, n,+-}^{f f}\left(r_{1}, \chi_{1}+\frac{\pi}{3}, r_{2}, \chi_{2}+\frac{\pi}{3}, \gamma-n \frac{\pi}{3}\right) \\
\exp \left(\sigma_{f} \frac{4 \pi i}{3}\right) \Psi_{\sigma, n,++}^{f f}\left(r_{1}, \chi_{1}+\frac{\pi}{3}, r_{2}, \chi_{2}+\frac{\pi}{3}, \gamma-n \frac{\pi}{3}\right)
\end{array}\right), \\
{ }^{R} \Psi_{\sigma, n}^{f f}\left(r_{1}, \chi_{1}, r_{2}, \chi_{2}, \gamma\right)= & \left(\begin{array}{c}
\Psi_{\sigma, n,++}^{f f}\left(r_{1},-\chi_{1}, r_{2},-\chi_{2},-\gamma\right) \\
\Psi_{\sigma, n,+-}^{f f}\left(r_{1},-\chi_{1}, r_{2},-\chi_{2},-\gamma\right) \\
\Psi_{\sigma, n,-+}^{f f}\left(r_{1},-\chi_{1}, r_{2},-\chi_{2},-\gamma\right) \\
\Psi_{\sigma, n,--}^{f f}\left(r_{1},-\chi_{1}, r_{2},-\chi_{2},-\gamma\right)
\end{array}\right) .
\end{aligned}
$$

Accordingly, the two-hole energy eigenstates transform as

$$
\begin{aligned}
{ }^{D_{i}} \Psi_{\sigma, m_{+}^{1}, m_{-}^{1}, m_{+}^{2}, m_{-}^{2}, m}^{f f}\left(r_{1}, \chi_{1}, r_{2}, \chi_{2}, \gamma\right) & =\exp \left(2 i k^{f} a_{i}\right) \Psi_{\sigma, m_{+}^{1}, m_{-}^{1}, m_{+}^{2}, m_{-}^{2}, m}^{f f}\left(r_{1}, \chi_{1}, r_{2}, \chi_{2}, \gamma\right), \\
{ }^{O^{\prime}} \Psi_{\sigma, m_{+}^{1}, m_{-}^{1}, m_{+}^{2}, m_{-}^{2}, m}^{\alpha \alpha}\left(r_{1}, \chi_{1}, r_{2}, \chi_{2}, \gamma\right) & =-\exp \left(i \sigma\left[m_{+}^{1}+m_{+}^{2}+n+\sigma-m n \frac{\pi}{3}\right)\right. \\
& \times \Psi_{-\sigma,-m_{-}^{1},-m_{+}^{1},-m_{-}^{2},-m_{+}^{2},-m}^{\beta \beta}\left(r_{1}, \chi_{1}, r_{2}, \chi_{2}, \gamma\right), \\
{ }^{O^{\prime}} \Psi_{\sigma, m_{+}^{1}, m_{-}^{1}, m_{+}^{2}, m_{-}^{2}, m}^{\beta \beta}\left(r_{1}, \chi_{1}, r_{2}, \chi_{2}, \gamma\right) & =-\exp \left(i \sigma\left[m_{+}^{1}+m_{+}^{2}+n-\sigma-m n\right] \frac{\pi}{3}\right) \\
& \times \Psi_{-\sigma,-m_{-}^{1},-m_{+}^{1},-m_{-}^{2},-m_{+}^{2},-m}^{\alpha \alpha}\left(r_{1}, \chi_{1}, r_{2}, \chi_{2}, \gamma\right), \\
{ }^{R} \Psi_{\sigma, m_{+}^{1}, m_{-}^{1}, m_{+}^{2}, m_{-}^{2}, m}^{\alpha \alpha}\left(r_{1}, \chi_{1}, r_{2}, \chi_{2}, \gamma\right) & =\Psi_{-\sigma, m_{+}^{1}, m_{-}^{1}, m_{+}^{2}, m_{-}^{2}, m}^{\beta \beta}\left(r_{1}, \chi_{1}, r_{2}, \chi_{2}, \gamma\right), \\
{ }^{R} \Psi_{\sigma, m_{+}^{1}, m_{-}^{1}, m_{+}^{2}, m_{-}^{2}, m}^{\beta \beta}\left(r_{1}, \chi_{1}, r_{2}, \chi_{2}, \gamma\right) & =\Psi_{-\sigma, m_{+}^{1}, m_{-}^{1}, m_{+}^{2}, m_{-}^{2}, m}^{\alpha \alpha}\left(r_{1}, \chi_{1}, r_{2}, \chi_{2}, \gamma\right) .
\end{aligned}
$$

Note that we have again used appropriate phase conventions for the radial wave function $\psi_{\sigma, m_{+}^{1}, m_{-}^{1}, m_{+}^{2}, m_{-}^{2}, m}\left(r_{1}, r_{2}\right)$. Regarding the rotation symmetries $O^{\prime}$, we have used

$$
\begin{aligned}
& \psi_{\sigma, m_{+}^{1}, m_{-}^{1}, m_{+}^{2}, m_{-}^{2}, m,--}^{f f}\left(r_{1}, r_{2}\right)=\psi_{-\sigma,-m_{-}^{1},-m_{+}^{1},-m_{-}^{2},-m_{+}^{2},-m,++}^{f^{\prime} f^{\prime}}\left(r_{1}, r_{2}\right), \\
& \psi_{\sigma, m_{+}^{1}, m_{-}^{1}, m_{+}^{2}, m_{-}^{2}, m,-+}^{f f}\left(r_{1}, r_{2}\right)=\psi_{-\sigma,-m_{-}^{1},-m_{+}^{1},-m_{-}^{2},-m_{+}^{2},-m,+-}^{f^{\prime} f^{\prime}}\left(r_{1}, r_{2}\right), \\
& \psi_{\sigma, m_{+}^{1}, m_{-}^{1}, m_{+}^{2}, m_{-}^{2}, m,+-}^{f f}\left(r_{1}, r_{2}\right)=\psi_{-\sigma,-m_{-}^{1},-m_{+}^{1},-m_{-}^{2},-m_{+}^{2},-m,-+}^{f^{\prime} f^{\prime}}\left(r_{1}, r_{2}\right), \\
& \psi_{\sigma, m_{+}^{1}, m_{-}^{1}, m_{+}^{2}, m_{-}^{2}, m,++}^{f f}\left(r_{1}, r_{2}\right)=\psi_{-\sigma,-m_{-}^{1},-m_{+}^{1},-m_{-}^{2},-m_{+}^{2},-m,--}^{f^{\prime} f^{\prime}}\left(r_{1}, r_{2}\right) .
\end{aligned}
$$


These relations are a consequence of the symmetries of the radial Schrödinger equation (4.37). With respect to the reflections $R$, we have used

$$
\begin{aligned}
& \psi_{\sigma, m_{+}^{1}, m_{-}^{1}, m_{+}^{2}, m_{-}^{2}, m,++}^{f f}\left(r_{1}, r_{2}\right)=\psi_{-\sigma, m_{+}^{1}, m_{-}^{1}, m_{+}^{2}, m_{-}^{2}, m,++}^{f^{\prime} f^{\prime}}\left(r_{1}, r_{2}\right), \\
& \psi_{\sigma, m_{+}^{1}, m_{-}^{1}, m_{+}^{2}, m_{-}^{2}, m,+-}^{f f}\left(r_{1}, r_{2}\right)=\psi_{-\sigma, m_{+}^{1}, m_{-}^{1}, m_{+}^{2}, m_{-}^{2}, m,+-}^{f^{\prime} f^{\prime}}\left(r_{1}, r_{2}\right), \\
& \psi_{\sigma, m_{+}^{1}, m_{-}^{1}, m_{+}^{2}, m_{-}^{2}, m,-+}^{f f}\left(r_{1}, r_{2}\right)=\psi_{-\sigma, m_{+}^{1}, m_{-}^{1}, m_{+}^{2}, m_{-}^{2}, m,-+}^{f^{\prime} f^{\prime}}\left(r_{1}, r_{2}\right), \\
& \psi_{\sigma, m_{+}^{1}, m_{-}^{1}, m_{+}^{2}, m_{-}^{2}, m,--}^{f f}\left(r_{1}, r_{2}\right)=\psi_{-\sigma, m_{+}^{1}, m_{-}^{1}, m_{+}^{2}, m_{-}^{2}, m,--}^{f^{\prime} f^{\prime}}\left(r_{1}, r_{2}\right) .
\end{aligned}
$$

The relations in Eq. (4.49) are a consequence of the symmetries of the radial Schrödinger equation (4.37) with $\Theta=0$. As before, in the case of $\Theta \neq 0$ or $\pi$, the reflection symmetry is explicitly broken by the Hopf term.

We now incorporate the Pauli principle and explicitly antisymmetrize the wave function in the artificial indices 1 and 2 , by acting with the pair permutation $P$,

$$
{ }^{P} \Psi_{\sigma, n}^{f f}\left(r_{1}, \chi_{1}, r_{2}, \chi_{2}, \gamma\right)=\left(\begin{array}{c}
\Psi_{\sigma, n,++}^{f f}\left(r_{2}, \chi_{2}, r_{1}, \chi_{1}, \gamma\right) \\
\Psi_{\sigma, n,-+}^{f f}\left(r_{2}, \chi_{2}, r_{1}, \chi_{1}, \gamma\right) \\
\Psi_{\sigma, n,+-}^{f f}\left(r_{2}, \chi_{2}, r_{1}, \chi_{1}, \gamma\right) \\
\Psi_{\sigma, n,--}^{f f}\left(r_{2}, \chi_{2}, r_{1}, \chi_{1}, \gamma\right)
\end{array}\right)
$$

For an energy eigenstate, we then have

$$
{ }^{P} \Psi_{\sigma, m_{+}^{1}, m_{-}^{1}, m_{+}^{2}, m_{-}^{2}, m}^{f f}\left(r_{1}, \chi_{1}, r_{2}, \chi_{2}, \gamma\right)=\Psi_{\sigma, m_{+}^{2}, m_{-}^{2}, m_{+}^{1}, m_{-}^{1}, m}^{f f}\left(r_{1}, \chi_{1}, r_{2}, \chi_{2}, \gamma\right)
$$

assuming a symmetric radial wave function,

$$
\begin{aligned}
& \psi_{\sigma, m_{+}^{1}, m_{-}^{1}, m_{+}^{2}, m_{-}^{2}, m,++}^{f f}\left(r_{2}, r_{1}\right)=\psi_{\sigma, m_{+}^{2}, m_{-}^{2}, m_{+}^{1}, m_{-}^{1}, m,++}^{f f}\left(r_{1}, r_{2}\right), \\
& \psi_{\sigma, m_{+}^{1}, m_{-}^{1}, m_{+}^{2}, m_{-}^{2}, m,-+}^{f f}\left(r_{2}, r_{1}\right)=\psi_{\sigma, m_{+}^{2}, m_{-}^{2}, m_{+}^{1}, m_{-}^{1}, m,+-}^{f f}\left(r_{1}, r_{2}\right), \\
& \psi_{\sigma, m_{+}^{1}, m_{-}^{1}, m_{+}^{2}, m_{-}^{2}, m,+-}^{f f}\left(r_{2}, r_{1}\right)=\psi_{\sigma, m_{+}^{2}, m_{-}^{2}, m_{+}^{1}, m_{-}^{1}, m,-+}^{f f}\left(r_{1}, r_{2}\right), \\
& \psi_{\sigma, m_{+}^{1}, m_{-}^{1}, m_{+}^{2}, m_{-}^{2}, m,--}^{f f}\left(r_{2}, r_{1}\right)=\psi_{\sigma, m_{+}^{2}, m_{-}^{2}, m_{+}^{1}, m_{-}^{1}, m,--}^{f f}\left(r_{1}, r_{2}\right) .
\end{aligned}
$$

The properly antisymmetrized wave function then amounts to

$$
\widetilde{\Psi}_{\sigma, n}^{f f}\left(r_{1}, \chi_{1}, r_{2}, \chi_{2}, \gamma\right)=\frac{1}{\sqrt{2}}\left[\Psi_{\sigma, n}^{f f}\left(r_{1}, \chi_{1}, r_{2}, \chi_{2}, \gamma\right)-{ }^{P} \Psi_{\sigma, n}^{f f}\left(r_{1}, \chi_{1}, r_{2}, \chi_{2}, \gamma\right)\right]
$$

implying that for an energy eigenstate, we have

$$
\begin{aligned}
& \widetilde{\Psi}_{\sigma, m_{+}^{1}, m_{-}^{1}, m_{+}^{2}, m_{-}^{2}, m}^{f f}\left(r_{1}, \chi_{1}, r_{2}, \chi_{2}, \gamma\right)= \\
& \frac{1}{\sqrt{2}}\left[\Psi_{\sigma, m_{+}^{1}, m_{-}^{1}, m_{+}^{2}, m_{-}^{2}, m}^{f f}\left(r_{1}, \chi_{1}, r_{2}, \chi_{2}, \gamma\right)-\Psi_{\sigma, m_{+}^{2}, m_{-}^{2}, m_{+}^{1}, m_{-}^{1}, m}^{f f}\left(r_{1}, \chi_{1}, r_{2}, \chi_{2}, \gamma\right)\right] .
\end{aligned}
$$


Note that the two sets of quantum numbers $m_{+}^{1}, m_{-}^{1}$ and $m_{+}^{2}, m_{-}^{2}$ must be different, in order to have a non-vanishing wave function. In the case of an antisymmetric radial wave function, one could allow $m_{+}^{1}=m_{+}^{2}$ and $m_{-}^{1}=m_{-}^{2}$.

Using Eq. (4.47), the transformation properties of the antisymmetrized two-hole energy eigenstates turn out to be

$$
\begin{aligned}
& D_{i} \widetilde{\Psi}_{\sigma, m_{+}^{1}, m_{-}^{1}, m_{+}^{2}, m_{-}^{2}, m}^{f f}\left(r_{1}, \chi_{1}, r_{2}, \chi_{2}, \gamma\right)=\exp \left(2 i k^{f} a_{i}\right) \widetilde{\Psi}_{\sigma, m_{+}^{1}, m_{-}^{1}, m_{+}^{2}, m_{-}^{2}, m}^{f f}\left(r_{1}, \chi_{1}, r_{2}, \chi_{2}, \gamma\right), \\
& O^{\prime} \widetilde{\Psi}_{\sigma, m_{+}^{1}, m_{-}^{1}, m_{+}^{2}, m_{-}^{2}, m}^{\alpha \alpha}\left(r_{1}, \chi_{1}, r_{2}, \chi_{2}, \gamma\right)=-\exp \left(i \sigma\left[m_{+}^{1}+m_{+}^{2}+n+\sigma-m n\right] \frac{\pi}{3}\right) \\
& \times \widetilde{\Psi}_{-\sigma,-m_{-}^{1},-m_{+}^{1},-m_{-}^{2},-m_{+}^{2},-m}^{\beta \beta}\left(r_{1}, \chi_{1}, r_{2}, \chi_{2}, \gamma\right), \\
& O^{\prime} \widetilde{\Psi}_{\sigma, m_{+}^{1}, m_{-}^{1}, m_{+}^{2}, m_{-}^{2}, m}^{\beta \beta}\left(r_{1}, \chi_{1}, r_{2}, \chi_{2}, \gamma\right)=-\exp \left(i \sigma\left[m_{+}^{1}+m_{+}^{2}+n-\sigma-m n\right] \frac{\pi}{3}\right) \\
& \times \widetilde{\Psi}_{-\sigma,-m_{-}^{1},-m_{+}^{1},-m_{-}^{2},-m_{+}^{2},-m}^{\alpha \alpha}\left(r_{1}, \chi_{1}, r_{2}, \chi_{2}, \gamma\right), \\
& R \widetilde{\Psi}_{\sigma, m_{+}^{1}, m_{-}^{1}, m_{+}^{2}, m_{-}^{2}, m}^{\alpha \alpha}\left(r_{1}, \chi_{1}, r_{2}, \chi_{2}, \gamma\right)=\widetilde{\Psi}_{-\sigma, m_{+}^{1}, m_{-}^{1}, m_{+}^{2}, m_{-}^{2}, m}^{\beta \beta}\left(r_{1}, \chi_{1}, r_{2}, \chi_{2}, \gamma\right), \\
& R \widetilde{\Psi}_{\sigma, m_{+}^{1}, m_{-}^{1}, m_{+}^{2}, m_{-}^{2}, m}^{\beta \beta}\left(r_{1}, \chi_{1}, r_{2}, \chi_{2}, \gamma\right)=\widetilde{\Psi}_{-\sigma, m_{+}^{1}, m_{-}^{1}, m_{+}^{2}, m_{-}^{2}, m}^{\alpha \alpha}\left(r_{1}, \chi_{1}, r_{2}, \chi_{2}, \gamma\right) \text {. }
\end{aligned}
$$

Finally, we take appropriate linear combinations of the states with flavors $\alpha \alpha$ and $\beta \beta$ in order to obtain eigenstates of $O^{\prime}$,

$$
\begin{gathered}
\widetilde{\Psi}_{\sigma, m_{+}^{1}, m_{-}^{1}, m_{+}^{2}, m_{-}^{2}, m}^{ \pm}\left(r_{1}, \chi_{1}, r_{2}, \chi_{2}, \gamma\right)=\frac{1}{\sqrt{2}}\left[\widetilde{\Psi}_{\sigma, m_{+}^{1}, m_{-}^{1}, m_{+}^{2}, m_{-}^{2}, m}^{\alpha \alpha}\left(r_{1}, \chi_{1}, r_{2}, \chi_{2}, \gamma\right)\right. \\
\left. \pm \widetilde{\Psi}_{-\sigma,-m_{-}^{1},-m_{+}^{1},-m_{-}^{2},-m_{+}^{2},-m}^{\beta \beta}\left(r_{1}, \chi_{1}, r_{2}, \chi_{2}, \gamma\right)\right],
\end{gathered}
$$

which transform as

$$
\begin{aligned}
O^{\prime} \widetilde{\Psi}_{\sigma, m_{+}^{1}, m_{-}^{1}, m_{+}^{2}, m_{-}^{2}, m}^{ \pm}\left(r_{1}, \chi_{1}, r_{2}, \chi_{2} \gamma\right) & =\mp \exp \left(i \sigma\left[m_{+}^{1}+m_{-}^{2}-m n\right] \frac{\pi}{3}\right) \\
& \times \widetilde{\Psi}_{\sigma, m_{+}^{1}, m_{-}^{1}, m_{+}^{2}, m_{-}^{2}, m}^{ \pm}\left(r_{1}, \chi_{1}, r_{2}, \chi_{2}, \gamma\right)
\end{aligned}
$$

We now systematically list all two-hole-Skyrmion wave functions $(n=1, m=0)$ that can be constructed with quantum numbers $m_{ \pm}^{i}$ up to \pm 2 . States with $s$-wave 
symmetry are

$$
\begin{aligned}
& \widetilde{\Psi}_{-, 2,2,1,1,0}^{+}\left(r_{1}, \chi_{1}, r_{2}, \chi_{2}, \gamma\right), \\
& \widetilde{\Psi}_{-, 1,1,2,2,0}^{+}\left(r_{1}, \chi_{1}, r_{2}, \chi_{2}, \gamma\right), \\
& \widetilde{\Psi}_{-,-2,-2,-1,-1,0}^{+}\left(r_{1}, \chi_{1}, r_{2}, \chi_{2}, \gamma\right), \\
& \widetilde{\Psi}_{-,-1,-1,-2,-2,0}^{+}\left(r_{1}, \chi_{1}, r_{2}, \chi_{2}, \gamma\right), \\
& \widetilde{\Psi}_{+,-2,0,0,2,0}^{-}\left(r_{1}, \chi_{1}, r_{2}, \chi_{2}, \gamma\right), \\
& \widetilde{\Psi}_{+, 0,2,-2,0,0}^{-}\left(r_{1}, \chi_{1}, r_{2}, \chi_{2}, \gamma\right), \\
& \widetilde{\Psi}_{-, 1,1,-1,-1,0}^{-}\left(r_{1}, \chi_{1}, r_{2}, \chi_{2}, \gamma\right), \\
& \widetilde{\Psi}_{-,-1,-1,1,1,0}^{-}\left(r_{1}, \chi_{1}, r_{2}, \chi_{2}, \gamma\right), \\
& \widetilde{\Psi}_{-, 2,2,-2,-2,0}^{-}\left(r_{1}, \chi_{1}, r_{2}, \chi_{2}, \gamma\right), \\
& \widetilde{\Psi}_{-,-2,-2,2,2,0}^{-}\left(r_{1}, \chi_{1}, r_{2}, \chi_{2}, \gamma\right) .
\end{aligned}
$$

States with $p$-wave symmetry read

$$
\begin{aligned}
& \widetilde{\Psi}_{+,-1,1,-2,0,0}^{-}\left(r_{1}, \chi_{1}, r_{2}, \chi_{2}, \gamma\right), \\
& \widetilde{\Psi}_{+,-2,0,-1,1,0}^{-}\left(r_{1}, \chi_{1}, r_{2}, \chi_{2}, \gamma\right), \\
& \widetilde{\Psi}_{+,-1,1,0,2,0}^{-}\left(r_{1}, \chi_{1}, r_{2}, \chi_{2}, \gamma\right), \\
& \widetilde{\Psi}_{+, 0,2,-1,1,0}^{-}\left(r_{1}, \chi_{1}, r_{2}, \chi_{2}, \gamma\right), \\
& \widetilde{\Psi}_{-, 0,0,-1,-1,0}^{-}\left(r_{1}, \chi_{1}, r_{2}, \chi_{2}, \gamma\right), \\
& \widetilde{\Psi}_{-, 0,0,1,1,0}^{-}\left(r_{1}, \chi_{1}, r_{2}, \chi_{2}, \gamma\right), \\
& \widetilde{\Psi}_{-,-1,-1,0,0,0}^{-}\left(r_{1}, \chi_{1}, r_{2}, \chi_{2}, \gamma\right), \\
& \widetilde{\Psi}_{-, 1,1,0,0,0}^{-}\left(r_{1}, \chi_{1}, r_{2}, \chi_{2}, \gamma\right), \\
& \widetilde{\Psi}_{-,-1,-1,2,2,0}^{-}\left(r_{1}, \chi_{1}, r_{2}, \chi_{2}, \gamma\right), \\
& \widetilde{\Psi}_{-, 2,2,-1,-1,0}^{-}\left(r_{1}, \chi_{1}, r_{2}, \chi_{2}, \gamma\right), \\
& \widetilde{\Psi}_{-,-2,-2,1,1,0}^{-}\left(r_{1}, \chi_{1}, r_{2}, \chi_{2}, \gamma\right), \\
& \widetilde{\Psi}_{-, 1,1,-2,-2,0}^{-}\left(r_{1}, \chi_{1}, r_{2}, \chi_{2}, \gamma\right) .
\end{aligned}
$$


States with $d$-wave symmetry correspond to

$$
\begin{aligned}
& \widetilde{\Psi}_{+,-1,1,-2,0,0}^{+}\left(r_{1}, \chi_{1}, r_{2}, \chi_{2}, \gamma\right), \\
& \widetilde{\Psi}_{+,-2,0,-1,1,0}^{+}\left(r_{1}, \chi_{1}, r_{2}, \chi_{2}, \gamma\right), \\
& \widetilde{\Psi}_{+,-1,1,0,2,0}^{+}\left(r_{1}, \chi_{1}, r_{2}, \chi_{2}, \gamma\right), \\
& \widetilde{\Psi}_{+, 0,2,-1,1,0}^{+}\left(r_{1}, \chi_{1}, r_{2}, \chi_{2}, \gamma\right), \\
& \widetilde{\Psi}_{-, 0,0,-1,-1,0}^{+}\left(r_{1}, \chi_{1}, r_{2}, \chi_{2}, \gamma\right), \\
& \widetilde{\Psi}_{-, 0,0,1,1,0}^{+}\left(r_{1}, \chi_{1}, r_{2}, \chi_{2}, \gamma\right), \\
& \widetilde{\Psi}_{-,-1,-1,0,0,0}^{+}\left(r_{1}, \chi_{1}, r_{2}, \chi_{2}, \gamma\right), \\
& \widetilde{\Psi}_{-, 1,1,0,0,0}^{+}\left(r_{1}, \chi_{1}, r_{2}, \chi_{2}, \gamma\right), \\
& \widetilde{\Psi}_{-,-1,-1,2,2,0}^{+}\left(r_{1}, \chi_{1}, r_{2}, \chi_{2}, \gamma\right), \\
& \widetilde{\Psi}_{-, 2,2,-1,-1,0}^{+}\left(r_{1}, \chi_{1}, r_{2}, \chi_{2}, \gamma\right), \\
& \widetilde{\Psi}_{-,-2,-2,1,1,0}^{+}\left(r_{1}, \chi_{1}, r_{2}, \chi_{2}, \gamma\right), \\
& \widetilde{\Psi}_{-, 1,1,-2,-2,0}^{+}\left(r_{1}, \chi_{1}, r_{2}, \chi_{2}, \gamma\right) .
\end{aligned}
$$

Finally, states with $f$-wave symmetry read

$$
\begin{aligned}
& \widetilde{\Psi}_{+,-2,0,0,2,0}^{+}\left(r_{1}, \chi_{1}, r_{2}, \chi_{2}, \gamma\right), \\
& \widetilde{\Psi}_{+, 0,2,-2,0,0}^{+}\left(r_{1}, \chi_{1}, r_{2}, \chi_{2}, \gamma\right), \\
& \widetilde{\Psi}_{-, 1,1,-1,-1,0}^{+}\left(r_{1}, \chi_{1}, r_{2}, \chi_{2}, \gamma\right), \\
& \widetilde{\Psi}_{-,-1,-1,1,1,0}^{+}\left(r_{1}, \chi_{1}, r_{2}, \chi_{2}, \gamma\right), \\
& \widetilde{\Psi}_{-, 2,2,-2,-2,0}^{+}\left(r_{1}, \chi_{1}, r_{2}, \chi_{2}, \gamma\right), \\
& \widetilde{\Psi}_{-,-2,-2,2,2,0}^{+}\left(r_{1}, \chi_{1}, r_{2}, \chi_{2}, \gamma\right), \\
& \widetilde{\Psi}_{-, 2,2,1,1,0}^{-}\left(r_{1}, \chi_{1}, r_{2}, \chi_{2}, \gamma\right), \\
& \widetilde{\Psi}_{-, 1,1,2,2,0}^{-}\left(r_{1}, \chi_{1}, r_{2}, \chi_{2}, \gamma\right), \\
& \widetilde{\Psi}_{-,-2,-2,-1,-1,0}^{-}\left(r_{1}, \chi_{1}, r_{2}, \chi_{2}, \gamma\right), \\
& \widetilde{\Psi}_{-,-1,-1,-2,-2,0}^{-}\left(r_{1}, \chi_{1}, r_{2}, \chi_{2}, \gamma\right) .
\end{aligned}
$$

Interestingly, as we have shown in Ref. [2], if one restricts oneself to the leading potentials that involve the $\Lambda$-term, one-magnon exchange can only occur between two holes of different flavor. In the same flavor case, this term merely leads to a contact interaction. Therefore there is nothing to be compared with on the magnonmediated bound-state side. It remains to be seen whether magnon-mediated binding is indeed possible if one goes beyond the leading $\Lambda$-term and how the symmetries of these bound states are related to those corresponding to the localization of two holes on a Skyrmion. Note that on the square lattice, the analogous $\Lambda$-term does lead to one-magnon exchange between holes of different as well as of the same flavor. Hence the formation of magnon-mediated two-hole bound states is possible in either case, in contrast to the honeycomb lattice. 


\section{Conclusions}

We have performed a careful symmetry analysis of the localization of doped holes on a topological Skyrmion defect in the staggered magnetization order parameter for an antiferromagnet on the honeycomb lattice. Our previous analysis for the square lattice had shown that two holes residing in two different hole pockets (and thus with different "flavors" $\alpha$ and $\beta$ ) naturally form a bound pair, which may qualify as a preformed Cooper pair candidate for high-temperature superconductivity. In the square lattice case, the most attractive channel for hole pair formation turned out to have the same quantum numbers as hole pairs bound by one-magnon exchange. In this work, we have seen that on the honeycomb lattice the situation is qualitatively different than on the square lattice. In particular, assuming the standard radial profile, a Skyrmion (antiSkyrmion) can only bind holes of flavor $\alpha(\beta)$. Hence, unlike on the square lattice, the formation of $\alpha \beta$-pairs is possible only for non-standard radial Skyrmion profiles. The question whether such profiles are energetically favorable, requires detailed numerical investigations of the dynamics. This goes beyond the symmetry analysis performed here, but provides an interesting topic for future studies. While understanding the dynamical mechanism responsible for high-temperature superconductivity remains extremely challenging, it seems promising to further investigate hole pair localization on topological Skyrmion defects. Our symmetry analysis, based on the systematic effective field theory for magnons and doped holes, provides a solid theoretical basis for future investigations.

\section{Acknowledgments}

C. P. H. and F.-J. J. thank the members of the Institute for Theoretical Physics at Bern University for warm hospitality. The present project has received funding from the Schweizerischer Nationalfonds as well as from the European Research Council by means of the European Union's Seventh Framework Programme (FP7/2007-2013)/ ERC grant agreement 339220.

\section{A Schrödinger Equation for Hole Pair with Differ- ent Flavor Localized on a Rotating Skyrmion}

In this appendix, we consider the analysis of bound states of two holes of different flavor localized on the same (anti-)Skyrmion. In contrast to two holes of the same flavor, the localization of a hole of flavor $\alpha$ and a second hole of flavor $\beta$ on the same (anti-)Skyrmion is not excluded by the Pauli principle. 
In the case of two holes of different flavor $\alpha$ and $\beta$, the Hamiltonian takes the form

$$
H=H^{\alpha}+H^{\beta}+H^{\gamma} .
$$

Here $H^{\alpha}$ and $H^{\beta}$ are the Hamiltonians for a hole of flavor $\alpha$ and $\beta$, respectively, given by

$$
\begin{aligned}
H^{\alpha} & =\left(\begin{array}{cccc}
H_{++}^{\alpha} & 0 & H_{+-}^{\alpha} & 0 \\
0 & H_{++}^{\alpha} & 0 & H_{+-}^{\alpha} \\
H_{-+}^{\alpha} & 0 & H_{--}^{\alpha} & 0 \\
0 & H_{-+}^{\alpha} & 0 & H_{--}^{\alpha}
\end{array}\right), \quad H^{\beta}=\left(\begin{array}{cccc}
H_{++}^{\beta} & H_{+-}^{\beta} & 0 & 0 \\
H_{-+}^{\beta} & H_{--}^{\beta} & 0 & 0 \\
0 & 0 & H_{++}^{\beta} & H_{+-}^{\beta} \\
0 & 0 & H_{-+}^{\beta} & H_{--}^{\beta}
\end{array}\right) \\
H^{\gamma} & =\left(\begin{array}{cccc}
H_{++++}^{\gamma} & 0 & 0 & 0 \\
0 & H_{+-+-}^{\gamma} & 0 & 0 \\
0 & 0 & H_{-+-+}^{\gamma} & 0 \\
0 & 0 & 0 & H_{----}^{\gamma}
\end{array}\right),
\end{aligned}
$$

with $H_{ \pm \pm}^{f}$ and $H_{ \pm \pm \pm \pm}^{\gamma}$ given in Eq.(4.35). For the two-hole energy eigenstate we make the ansatz

$$
\begin{aligned}
& \Psi_{\sigma, m_{+}^{\alpha}, m_{-}^{\alpha}, m_{+}^{\beta}, m_{-}^{\beta}, m^{\alpha}}^{\alpha \beta}\left(r_{\alpha}, \chi_{\alpha}, r_{\beta}, \chi_{\beta}, \gamma\right)= \\
& \left(\begin{array}{c}
\psi_{\sigma, m_{+}^{\alpha}, m_{-}^{\alpha}, m_{+}^{\beta}, m_{-}^{\beta}, m_{,++}}^{\alpha \beta}\left(r_{\alpha}, r_{\beta}\right) \exp \left(i \sigma\left[m_{+}^{\alpha} \chi_{\alpha}+m_{+}^{\beta} \chi_{\beta}\right]\right) \exp (i \sigma(m-1) \gamma) \\
\psi_{\sigma, m_{+}^{\alpha}, m_{-}^{\alpha}, m_{+}^{\beta}, m_{-}^{\beta}, m,+-}^{\alpha,}\left(r_{\alpha}, r_{\beta}\right) \exp \left(i \sigma\left[m_{+}^{\alpha} \chi_{\alpha}+m_{-}^{\beta} \chi_{\beta}\right]\right) \exp (i \sigma m \gamma) \\
\psi_{\sigma, m_{+}^{\alpha}, m_{-}^{\alpha}, m_{+}^{\beta}, m_{-}^{\beta}, m,-+}^{\alpha \beta}\left(r_{\alpha}, r_{\beta}\right) \exp \left(i \sigma\left[m_{-}^{\alpha} \chi_{\alpha}+m_{+}^{\beta} \chi_{\beta}\right]\right) \exp (i \sigma m \gamma) \\
\psi_{\sigma, m_{+}^{\alpha}, m_{-}^{\alpha}, m_{+}^{\beta}, m_{-}^{\beta}, m_{,--}}^{\alpha \beta}\left(r_{\alpha}, r_{\beta}\right) \exp \left(i \sigma\left[m_{-}^{\alpha} \chi_{\alpha}+m_{-}^{\beta} \chi_{\beta}\right]\right) \exp (i \sigma(m+1) \gamma)
\end{array}\right) .
\end{aligned}
$$

Recall that the Schrödinger equation is only satisfied if $m_{-}^{f}-m_{+}^{f}=n+\sigma \sigma_{f}$. As for the Skyrmion with no holes localized on it, here $m$ is again an integer. Accordingly, the radial Schrödinger equation amounts to

$$
H_{r} \psi_{\sigma, m_{+}^{\alpha}, m_{-}^{\alpha}, m_{+}^{\beta}, m_{-}^{\beta}, m}^{\alpha \beta}\left(r_{\alpha}, r_{\beta}\right)=E_{\sigma, m_{+}^{\alpha}, m_{-}^{\alpha}, m_{+}^{\beta}, m_{-}^{\beta}, m} \psi_{\sigma, m_{+}^{\alpha}, m_{-}^{\alpha}, m_{+}^{\beta}, m_{-}^{\beta}, m}^{\alpha \beta}\left(r_{\alpha}, r_{\beta}\right),
$$

with

$$
\psi_{\sigma, m_{+}^{\alpha}, m_{-}^{\alpha}, m_{+}^{\beta}, m_{-}^{\beta}, m}^{\alpha \beta}\left(r_{\alpha}, r_{\beta}\right)=\left(\begin{array}{c}
\psi_{\sigma, m_{+}^{\alpha}, m_{-}^{\alpha}, m_{+}^{\beta}, m_{-}^{\beta}, m,++}^{\alpha \beta}\left(r_{\alpha}, r_{\beta}\right) \\
\psi_{\sigma, m_{+}^{\alpha}, m_{-}^{\alpha}, m_{+}^{\beta}, m_{-}^{\beta}, m,+-}^{\alpha \beta}\left(r_{\alpha}, r_{\beta}\right) \\
\psi_{\sigma, m_{+}^{\alpha}, m_{-}^{\alpha}, m_{+}^{\beta}, m_{-}^{\beta}, m,-+}^{\alpha \beta}\left(r_{\alpha}, r_{\beta}\right) \\
\psi_{\sigma, m_{+}^{\alpha}, m_{-}^{\alpha}, m_{+}^{\beta}, m_{-}^{\beta}, m,--}^{\alpha \beta}\left(r_{\alpha}, r_{\beta}\right)
\end{array}\right) .
$$

The various terms in the radial Hamiltonian

$$
H_{r}=H_{r}^{\alpha}+H_{r}^{\beta}+H_{r}^{\gamma}
$$


take the explicit form

$$
\begin{aligned}
H_{r}^{\alpha}= & \left(\begin{array}{cccc}
H_{r++}^{\alpha} & 0 & H_{r+-}^{\alpha} & 0 \\
0 & H_{r++}^{\alpha} & 0 & H_{r+-}^{\alpha} \\
H_{r-+}^{\alpha} & 0 & H_{r--}^{\alpha} & 0 \\
0 & H_{r-+}^{\alpha} & 0 & H_{r--}^{\alpha}
\end{array}\right), \\
H_{r}^{\beta}= & \left(\begin{array}{cccc}
H_{r++}^{\beta} & H_{r+-}^{\beta} & 0 & 0 \\
H_{r-+}^{\beta} & H_{r--}^{\beta} & 0 & 0 \\
0 & 0 & H_{r++}^{\beta} & H_{r+-}^{\beta} \\
0 & 0 & H_{r-+}^{\beta} & H_{r--}^{\beta}
\end{array}\right), \\
H_{r}^{\gamma}= & \left(\begin{array}{cccc}
H_{r++++}^{\gamma} & 0 & 0 & 0 \\
0 & H_{r+-+-}^{\gamma} & 0 & 0 \\
0 & 0 & H_{r-+-+}^{\gamma} & 0 \\
0 & 0 & 0 & H_{r----}^{\gamma}
\end{array}\right) .
\end{aligned}
$$

The corresponding matrix elements of the fermionic part of the radial Hamiltonian read

$$
\begin{aligned}
& H_{r++}^{f}=-\frac{1}{2 M^{\prime}}\left[\partial_{r_{f}}^{2}+\frac{1}{r_{f}} \partial_{r_{f}}-\frac{1}{r_{f}^{2}}\left(m_{+}^{f}+\frac{n}{2}\left(1-f\left(r_{f}\right)\right)\right)^{2}\right], \\
& H_{r+-}^{f}=H_{r-+}^{f}=\frac{\Lambda}{2}\left[\frac{f^{\prime}\left(r_{f}\right)}{\sqrt{1-f^{2}\left(r_{f}\right)}}+\frac{\sigma \sigma_{f} n}{r_{f}} \sqrt{1-f^{2}\left(r_{f}\right)}\right], \\
& H_{r--}^{f}=-\frac{1}{2 M^{\prime}}\left[\partial_{r_{f}}^{2}+\frac{1}{r_{f}} \partial_{r_{f}}-\frac{1}{r_{f}^{2}}\left(m_{-}^{f}-\frac{n}{2}\left(1-f\left(r_{f}\right)\right)\right)^{2}\right],
\end{aligned}
$$

while the contributions describing the rotating Skyrmion amount to

$$
\begin{aligned}
& H_{r++++}^{\gamma}=\frac{n^{2}}{2 \mathcal{D}(\rho) \rho^{2}}\left(m-1+\sigma n \frac{\Theta}{2 \pi}-\frac{1}{2}\left(1-f\left(r_{\alpha}\right)\right)-\frac{1}{2}\left(1-f\left(r_{\beta}\right)\right)\right)^{2}, \\
& H_{r+-+-}^{\gamma}=\frac{n^{2}}{2 \mathcal{D}(\rho) \rho^{2}}\left(m+\sigma n \frac{\Theta}{2 \pi}-\frac{1}{2}\left(1-f\left(r_{\alpha}\right)\right)+\frac{1}{2}\left(1-f\left(r_{\beta}\right)\right)\right)^{2}, \\
& H_{r-+-+}^{\gamma}=\frac{n^{2}}{2 \mathcal{D}(\rho) \rho^{2}}\left(m+\sigma n \frac{\Theta}{2 \pi}+\frac{1}{2}\left(1-f\left(r_{\alpha}\right)\right)-\frac{1}{2}\left(1-f\left(r_{\beta}\right)\right)\right)^{2} \\
& H_{r----}^{\gamma}=\frac{n^{2}}{2 \mathcal{D}(\rho) \rho^{2}}\left(m+1+\sigma n \frac{\Theta}{2 \pi}+\frac{1}{2}\left(1-f\left(r_{\alpha}\right)\right)+\frac{1}{2}\left(1-f\left(r_{\beta}\right)\right)\right)^{2} .
\end{aligned}
$$




\section{B Hole Pair of Different Flavor Localized on a Skyrmion: Symmetry Properties}

The spin operator I, Eq. (4.43), satisfies

$$
\begin{aligned}
& I \Psi_{\sigma, m_{+}^{\alpha}, m_{-}^{\alpha}, m_{+}^{\beta}, m_{-}^{\beta}, m}^{\alpha \beta}\left(r_{\alpha}, \chi_{\alpha}, r_{\beta}, \chi_{\beta}, \gamma\right)= \\
& \left(m+\sigma n \frac{\Theta}{2 \pi}\right) \Psi_{\sigma, m_{+}^{\alpha}, m_{-}^{\alpha}, m_{+}^{\beta}, m_{-}^{\beta}, m}^{\alpha \beta}\left(r_{\alpha}, \chi_{\alpha}, r_{\beta}, \chi_{\beta}, \gamma\right) .
\end{aligned}
$$

Since $m$ takes integer values, at least for $\Theta=0$, the state containing two holes of different flavor localized on a Skyrmion has integer spin as well.

Under the symmetries $D_{i}, O^{\prime}$, and $R$, the general two-hole wave function

$$
\Psi_{\sigma, n}^{\alpha \beta}\left(r_{\alpha}, \chi_{\alpha}, r_{\beta}, \chi_{\beta}, \gamma\right)=\left(\begin{array}{c}
\Psi_{\sigma, n,++}^{\alpha \beta}\left(r_{\alpha}, \chi_{\alpha}, r_{\beta}, \chi_{\beta}, \gamma\right) \\
\Psi_{\sigma, n,+-}^{\alpha \beta}\left(r_{\alpha}, \chi_{\alpha}, r_{\beta}, \chi_{\beta}, \gamma\right) \\
\Psi_{\sigma, n,-+}^{\alpha \beta}\left(r_{\alpha}, \chi_{\alpha}, r_{\beta}, \chi_{\beta}, \gamma\right) \\
\Psi_{\sigma, n,--}^{\alpha \beta}\left(r_{\alpha}, \chi_{\alpha}, r_{\beta}, \chi_{\beta}, \gamma\right)
\end{array}\right)
$$

transforms as

$$
\begin{aligned}
{ }^{D_{i}} \Psi_{\sigma, n}^{\alpha \beta}\left(r_{\alpha}, \chi_{\alpha}, r_{\beta}, \chi_{\beta}, \gamma\right)= & \exp \left(i\left(k^{\alpha}+k^{\beta}\right) a_{i}\right)\left(\begin{array}{c}
\Psi_{\sigma, n,++}^{\alpha \beta}\left(r_{\alpha}, \chi_{\alpha}, r_{\beta}, \chi_{\beta}, \gamma\right) \\
\Psi_{\sigma, n,+-}^{\alpha \beta}\left(r_{\alpha}, \chi_{\alpha}, r_{\beta}, \chi_{\beta}, \gamma\right) \\
\Psi_{\sigma, n,-+}^{\alpha \beta}\left(r_{\alpha}, \chi_{\alpha}, r_{\beta}, \chi_{\beta}, \gamma\right) \\
\Psi_{\sigma, n,--}^{\alpha \beta}\left(r_{\alpha}, \chi_{\alpha}, r_{\beta}, \chi_{\beta}, \gamma\right)
\end{array}\right), \\
O^{\prime} \Psi_{\sigma, n}^{\alpha \beta}\left(r_{\alpha}, \chi_{\alpha}, r_{\beta}, \chi_{\beta}, \gamma\right)= & \left(\begin{array}{c}
\Psi_{\sigma, n,--}^{\alpha \beta}\left(r_{\beta}, \chi_{\beta}+\frac{\pi}{3}, r_{\alpha}, \chi_{\alpha}+\frac{\pi}{3}, \gamma-n \frac{\pi}{3}\right) \\
-\exp \left(\frac{4 \pi i}{3}\right) \Psi_{\sigma, n,+-}^{\alpha \beta}\left(r_{\beta}, \chi_{\beta}+\frac{\pi}{3}, r_{\alpha}, \chi_{\alpha}+\frac{\pi}{3}, \gamma-n \frac{\pi}{3}\right) \\
-\exp \left(-\frac{4 \pi i}{3}\right) \Psi_{\sigma, n,-+}^{\alpha \beta}\left(r_{\beta}, \chi_{\beta}+\frac{\pi}{3}, r_{\alpha}, \chi_{\alpha}+\frac{\pi}{3}, \gamma-n \frac{\pi}{3}\right) \\
\Psi_{\sigma, n,++}^{\alpha \beta}\left(r_{\beta}, \chi_{\beta}+\frac{\pi}{3}, r_{\alpha}, \chi_{\alpha}+\frac{\pi}{3}, \gamma-n \frac{\pi}{3}\right)
\end{array}\right), \\
{ }^{R} \Psi_{\sigma, n}^{\alpha \beta}\left(r_{\alpha}, \chi_{\alpha}, r_{\beta}, \chi_{\beta}, \gamma\right)= & \left(\begin{array}{c}
\Psi_{\sigma, n,++}^{\alpha \beta}\left(r_{\beta},-\chi_{\beta}, r_{\alpha},-\chi_{\alpha},-\gamma\right) \\
\Psi_{\sigma, n,-+}^{\alpha \beta}\left(r_{\beta},-\chi_{\beta}, r_{\alpha},-\chi_{\alpha},-\gamma\right) \\
\Psi_{\sigma, n,+-}^{\alpha \beta}\left(r_{\beta},-\chi_{\beta}, r_{\alpha},-\chi_{\alpha},-\gamma\right) \\
\Psi_{\sigma, n,--}^{\alpha \beta}\left(r_{\beta},-\chi_{\beta}, r_{\alpha},-\chi_{\alpha},-\gamma\right)
\end{array}\right) .
\end{aligned}
$$

One readily derives the following transformation properties of the two-hole energy eigenstates,

$$
\begin{aligned}
D_{i} \Psi_{\sigma, m_{+}^{\alpha}, m_{-}^{\alpha}, m_{+}^{\beta}, m_{-}^{\beta}, m}^{\alpha \beta}\left(r_{\alpha}, \chi_{\alpha}, r_{\beta}, \chi_{\beta}, \gamma\right) & =\Psi_{\sigma, m_{+}^{\alpha}, m_{-}^{\alpha}, m_{+}^{\beta}, m_{-}^{\beta}, m}^{\alpha \beta}\left(r_{\alpha}, \chi_{\alpha}, r_{\beta}, \chi_{\beta}, \gamma\right), \\
{ }^{\prime} \Psi_{\sigma, m_{+}^{\alpha}, m_{-}^{\alpha}, m_{+}^{\beta}, m_{-}^{\beta}, m}^{\alpha \beta}\left(r_{\alpha}, \chi_{\alpha}, r_{\beta}, \chi_{\beta}, \gamma\right) & =\exp \left(i \sigma\left[m_{+}^{\alpha}+m_{-}^{\beta}+\sigma-m n\right] \frac{\pi}{3}\right) \\
& \times \Psi_{-\sigma,-m_{-}^{\beta},-m_{+}^{\beta},-m_{-}^{\alpha},-m_{+}^{\alpha},-m}^{\alpha \beta}\left(r_{\alpha}, \chi_{\alpha}, r_{\beta}, \chi_{\beta}, \gamma\right), \\
{ }^{R} \Psi_{\sigma, m_{+}^{\alpha}, m_{-}^{\alpha}, m_{+}^{\beta}, m_{-}^{\beta}, m}^{\alpha \beta}\left(r_{\alpha}, \chi_{\alpha}, r_{\beta}, \chi_{\beta}, \gamma\right) & =\Psi_{-\sigma, m_{+}^{\beta}, m_{-}^{\beta}, m_{+}^{\alpha}, m_{-}^{\alpha}, m}^{\alpha \beta}\left(r_{\alpha}, \chi_{\alpha}, r_{\beta}, \chi_{\beta}, \gamma\right) .
\end{aligned}
$$


Note that we have assumed appropriate phase conventions for the radial wave function $\psi_{\sigma, m_{+}^{\alpha}, m_{-}^{\alpha}, m_{+}^{\beta}, m_{-}^{\beta}, m}\left(r_{\alpha}, r_{\beta}\right)$, which follow from the symmetries of the radial Schrödinger equation (A.4). Explicitly, for the rotation symmetry $O^{\prime}$ we have used

$$
\begin{aligned}
& \psi_{\sigma, m_{+}^{\alpha}, m_{-}^{\alpha}, m_{+}^{\beta}, m_{-}^{\beta}, m,--}^{\alpha \beta}\left(r_{\beta}, r_{\alpha}\right)=\psi_{-\sigma,-m_{-}^{\beta},-m_{+}^{\beta},-m_{-}^{\alpha},-m_{+}^{\alpha},-m,++}^{\alpha \beta}\left(r_{\alpha}, r_{\beta}\right), \\
& \psi_{\sigma, m_{+}^{\alpha \beta}, m_{-}^{\alpha}, m_{+}^{\beta}, m_{-}^{\beta}, m,+-}^{\alpha \beta}\left(r_{\beta}, r_{\alpha}\right)=\psi_{-\sigma,-m_{-}^{\beta},-m_{+}^{\beta},-m_{-}^{\alpha},-m_{+}^{\alpha},-m,+-}^{\alpha \beta}\left(r_{\alpha}, r_{\beta}\right), \\
& \psi_{\sigma, m_{+}^{\alpha}, m_{-}^{\alpha}, m_{+}^{\beta}, m_{-}^{\beta}, m,-+}^{\alpha \beta}\left(r_{\beta}, r_{\alpha}\right)=\psi_{-\sigma,-m_{-}^{\beta},-m_{+}^{\beta},-m_{-}^{\alpha},-m_{+}^{\alpha},-m,-+}^{\alpha \beta}\left(r_{\alpha}, r_{\beta}\right), \\
& \psi_{\sigma, m_{+}^{\alpha}, m_{-}^{\alpha}, m_{+}^{\beta}, m_{-}^{\beta}, m,++}^{\alpha \beta}\left(r_{\beta}, r_{\alpha}\right)=\psi_{-\sigma,-m_{-}^{\alpha},-m_{+}^{\beta},-m_{-}^{\alpha},-m_{+}^{\alpha},-m,--}^{\alpha \beta}\left(r_{\alpha}, r_{\beta}\right) .
\end{aligned}
$$

For the reflection symmetry $R$ we have used

$$
\begin{aligned}
& \psi_{\sigma, m_{+}^{\alpha}, m_{-}^{\alpha}, m_{+}^{\beta}, m_{-}^{\beta}, m,++}^{\alpha \beta}\left(r_{\beta}, r_{\alpha}\right)=\psi_{-\sigma, m_{+}^{\beta}, m_{-}^{\beta}, m_{+}^{\alpha}, m_{-}^{\alpha}, m,++}^{\alpha \beta}\left(r_{\alpha}, r_{\beta}\right), \\
& \psi_{\sigma, m_{+}^{\alpha}, m_{-}^{\alpha}, m_{+}^{\beta}, m_{-}^{\beta}, m,-+}^{\alpha \beta}\left(r_{\beta}, r_{\alpha}\right)=\psi_{-\sigma, m_{+}^{\beta}, m_{-}^{\beta}, m_{+}^{\alpha}, m_{-}^{\alpha}, m,+-}^{\alpha \beta}\left(r_{\alpha}, r_{\beta}\right), \\
& \psi_{\sigma, m_{+}^{\alpha}, m_{-}^{\alpha}, m_{+}^{\beta}, m_{-}^{\beta}, m,+-}^{\alpha \beta}\left(r_{\beta}, r_{\alpha}\right)=\psi_{-\sigma, m_{+}^{\beta}, m_{-}^{\beta}, m_{+}^{\alpha}, m_{-}^{\alpha}, m,-+}^{\alpha \beta}\left(r_{\alpha}, r_{\beta}\right), \\
& \psi_{\sigma, m_{+}^{\alpha}, m_{-}^{\alpha}, m_{+}^{\beta}, m_{-}^{\beta}, m,--}^{\alpha \beta}\left(r_{\beta}, r_{\alpha}\right)=\psi_{-\sigma, m_{+}^{\beta}, m_{-}^{\beta}, m_{+}^{\alpha}, m_{-}^{\alpha}, m,--}^{\alpha \beta}\left(r_{\alpha}, r_{\beta}\right) .
\end{aligned}
$$

Again, the relations in Eq.(B.6) are a consequence of the symmetries of the radial Schrödinger equation (A.4) for $\Theta=0$. For $\Theta \neq 0$ or $\pi$, the reflection symmetry is explicitly broken by the Hopf term.

We now take appropriate linear combinations of Skyrmion and anti-Skyrmion states which are eigenstates of the combined rotation $O^{\prime}$,

$$
\begin{gathered}
\Psi_{\sigma, m_{+}^{\alpha}, m_{-}^{\alpha}, m_{+}^{\beta}, m_{-}^{\beta}, m}^{ \pm}\left(r_{\alpha}, \chi_{\alpha}, r_{\beta}, \chi_{\beta}, \gamma\right)=\frac{1}{\sqrt{2}}\left[\Psi_{\sigma, m_{+}^{\alpha}, m_{-}^{\alpha}, m_{+}^{\beta}, m_{-}^{\beta}, m}^{\alpha \beta}\left(r_{\alpha}, \chi_{\alpha}, r_{\beta}, \chi_{\beta}, \gamma\right)\right. \\
\left. \pm \Psi_{-\sigma,-m_{-}^{\beta},-m_{+}^{\beta},-m_{-}^{\alpha},-m_{+}^{\alpha},-m}^{\alpha \beta}\left(r_{\alpha}, \chi_{\alpha}, r_{\beta}, \chi_{\beta}, \gamma\right)\right] .
\end{gathered}
$$

Under the various symmetries, these states transform as

$$
\begin{aligned}
{ }^{D_{i}} \Psi_{\sigma, m_{+}^{\alpha}, m_{-}^{\alpha}, m_{+}^{\beta}, m_{-}^{\beta}, m}^{ \pm}\left(r_{\alpha}, \chi_{\alpha}, r_{\beta}, \chi_{\beta}, \gamma\right) & =\Psi_{\sigma, m_{+}^{\alpha}, m_{-}^{\alpha}, m_{+}^{\beta}, m_{-}^{\beta}, m}^{ \pm}\left(r_{\alpha}, \chi_{\alpha}, r_{\beta}, \chi_{\beta}, \gamma\right), \\
{ }^{O^{\prime}} \Psi_{\sigma, m_{+}^{\alpha}, m_{-}^{\alpha}, m_{+}^{\beta}, m_{-}^{\beta}, m}^{ \pm}\left(r_{\alpha}, \chi_{\alpha}, r_{\beta}, \chi_{\beta}, \gamma\right) & = \pm \exp \left(i \sigma\left[m_{+}^{\alpha}+m_{-}^{\beta}+\sigma-m n\right] \frac{\pi}{3}\right) \\
& \times \Psi_{\sigma, m_{+}^{\alpha}, m_{-}^{\alpha}, m_{+}^{\beta}, m_{-}^{\beta}, m}^{ \pm}\left(r_{\alpha}, \chi_{\alpha}, r_{\beta}, \chi_{\beta}, \gamma\right), \\
{ }^{R} \Psi_{\sigma, m_{+}^{\alpha}, m_{-}^{\alpha}, m_{+}^{\beta}, m_{-}^{\beta}, m}^{ \pm}\left(r_{\alpha}, \chi_{\alpha}, r_{\beta}, \chi_{\beta}, \gamma\right) & = \pm \Psi_{\sigma,-m_{-}^{ \pm},-m_{+}^{\alpha},-m_{-}^{\beta},-m_{+}^{\beta},-m}^{\text {(B. }}\left(r_{\alpha}, \chi_{\alpha}, r_{\beta}, \chi_{\beta}, \gamma\right) .
\end{aligned}
$$




\section{Comparison with States of Two Holes Bound by One-Magnon Exchange}

While we have considered the localization of two holes of different flavor on a Skyrmion in the previous section, it is instructive to compare this with the formation of twohole bound states mediated by one-magnon exchange, discussed in Ref. [2]. In what follows, we first summarize these results.

In the rest frame, the Schrödinger equation describing the relative motion of two holes with different flavors $\alpha$ and $\beta$, takes the form

$$
\left(\begin{array}{cc}
-\frac{1}{M^{\prime}} \Delta & \gamma \frac{1}{\vec{r}^{2}} \exp (-2 i \varphi) \\
\gamma \frac{1}{\vec{r}^{2}} \exp (2 i \varphi) & -\frac{1}{M^{\prime}} \Delta
\end{array}\right)\left(\begin{array}{l}
\Psi_{1}(\vec{r}) \\
\Psi_{2}(\vec{r})
\end{array}\right)=E\left(\begin{array}{c}
\Psi_{1}(\vec{r}) \\
\Psi_{2}(\vec{r})
\end{array}\right),
$$

where

$$
\gamma=\frac{\Lambda^{2}}{2 \pi \rho_{s}}
$$

The two probability amplitudes $\Psi_{1}(\vec{r})$ and $\Psi_{2}(\vec{r})$ refer to the two spin-flavor combinations $\alpha_{+} \beta_{-}$and $\alpha_{-} \beta_{+}$, respectively, with the distance vector $\vec{r}$ pointing from the $\beta$ to the $\alpha$ hole. Note that the holes undergo a spin flip during the one-magnon exchange process and that we are dealing with a two-component Schrödinger equation describing the relative motion of the hole pair.

With the ansatz

$$
\Psi_{1}(r, \varphi)=R_{1}(r) \exp \left(i m_{1} \varphi\right), \quad \Psi_{2}(r, \varphi)=R_{2}(r) \exp \left(i m_{2} \varphi\right),
$$

the radial and the angular part can be separated if the angular quantum numbers obey the condition $m_{2}-m_{1}=2$. Using the parameter $\tilde{m}$,

$$
m_{1}=\tilde{m}-1, \quad m_{2}=\tilde{m}+1,
$$

the radial equations amount to

$$
\begin{aligned}
& -\left(\frac{d^{2}}{d r^{2}}+\frac{1}{r} \frac{d}{d r}-\frac{1}{r^{2}}(\tilde{m}-1)^{2}\right) R_{1}(r)+\gamma M^{\prime} \frac{R_{2}(r)}{r^{2}}=M^{\prime} E R_{1}(r), \\
& -\left(\frac{d^{2}}{d r^{2}}+\frac{1}{r} \frac{d}{d r}-\frac{1}{r^{2}}(\tilde{m}+1)^{2}\right) R_{2}(r)+\gamma M^{\prime} \frac{R_{1}(r)}{r^{2}}=M^{\prime} E R_{2}(r) .
\end{aligned}
$$

In the particular case $\tilde{m}=0$, the set of equations reduces to

$$
\left[-\left(\frac{d^{2}}{d r^{2}}+\frac{1}{r} \frac{d}{d r}\right)+\left(1-\gamma M^{\prime}\right) \frac{1}{r^{2}}\right] R(r)=-M^{\prime}|E| R(r),
$$

with $R(r)=R_{1}(r)-R_{2}(r)$, which is solved analytically by a modified Bessel function

$$
R(r)=A K_{\nu}\left(\sqrt{M^{\prime}|E|} r\right), \quad \nu=i \sqrt{\gamma M^{\prime}-1} .
$$


We have modeled the short-range repulsion between the two holes with a hard-core radius $r_{0}$ by requiring $R\left(r_{0}\right)=0$ for $r \leq r_{0}$. The potential in the radial equation (C.6) is negative and thus attractive, provided that the low-energy constants satisfy the relation

$$
1-\frac{M^{\prime} \Lambda^{2}}{2 \pi \rho_{s}} \leq 0
$$

Accordingly, $\Lambda$ must be larger than the critical value

$$
\Lambda_{c}=\sqrt{\frac{2 \pi \rho_{s}}{M^{\prime}}}
$$

in order for a magnon-mediated bound state to occur. The energy of the $n$-th excited bound state amounts to

$$
E_{n} \sim-\frac{1}{M^{\prime} r_{0}^{2}} \exp \left(\frac{-2 \pi n}{\sqrt{\gamma M^{\prime}-1}}\right)
$$

On the other hand, the angular part of the ground-state wave equation $(\tilde{m}=0)$ of two holes of flavors $\alpha$ and $\beta$ takes the simple form

$$
\Psi(r, \varphi)=\left(\begin{array}{c}
\Psi_{1}(\vec{r}) \\
\Psi_{2}(\vec{r})
\end{array}\right)=R(r)\left(\begin{array}{c}
\exp (-i \varphi) \\
-\exp (i \varphi)
\end{array}\right) .
$$

While the ground state wave function is invariant under reflections $R$, shifts $D_{i}$, and accidental continuous rotations $O(\gamma)$, under the combined rotation $O^{\prime}$, it picks up a sign,

$$
{ }^{\prime} \Psi(r, \varphi)=-\Psi(r, \varphi) .
$$

Although the probability distribution of the ground state seems to imply $s$-wave symmetry (see Ref. [2], Fig.6), we are in fact dealing with $f$-wave symmetry.

Let us now compare magnon-mediated hole binding with the localization of two holes on a Skyrmion. In the case of a rotating Skyrmion with $n=1$ and $m=0$, it is possible to construct a variety of two-hole-Skyrmion wave functions with small quantum numbers. In the following we systematically enumerate $s^{-}, p-, d$ - and $f$-wave states of the wave function, corresponding to quantum numbers $m_{ \pm}^{\alpha}, m_{ \pm}^{\beta}$ up to \pm 2 . We first consider the linear combinations

$$
\Psi_{\sigma, m_{+}^{\alpha}, m_{-}^{\alpha}, m_{+}^{\beta}, m_{-}^{\beta}, m}^{+}\left(r_{\alpha}, \chi_{\alpha}, r_{\beta}, \chi_{\beta}, \gamma\right)
$$

defined in Eq. (B.7). States with $s$-wave symmetry are

$$
\begin{aligned}
& \Psi_{+,-1,1,0,0,0}^{+}\left(r_{\alpha}, \chi_{\alpha}, r_{\beta}, \chi_{\beta}, \gamma\right), \\
& \Psi_{+, 0,2,-1,-1,0}^{+}\left(r_{\alpha}, \chi_{\alpha}, r_{\beta}, \chi_{\beta}, \gamma\right), \\
& \Psi_{+,-2,0,1,1,0}^{+}\left(r_{\alpha}, \chi_{\alpha}, r_{\beta}, \chi_{\beta}, \gamma\right), \\
& \Psi_{-, 0,0,-1,1,0}^{+}\left(r_{\alpha}, \chi_{\alpha}, r_{\beta}, \chi_{\beta}, \gamma\right), \\
& \Psi_{-, 1,1,-2,0,0}^{+}\left(r_{\alpha}, \chi_{\alpha}, r_{\beta}, \chi_{\beta}, \gamma\right), \\
& \Psi_{-,-1,-1,0,2,0}^{+}\left(r_{\alpha}, \chi_{\alpha}, r_{\beta}, \chi_{\beta}, \gamma\right) .
\end{aligned}
$$


States with $p$-wave symmetry read

$$
\begin{aligned}
& \Psi_{+,-1,1,1,1,0}^{+}\left(r_{\alpha}, \chi_{\alpha}, r_{\beta}, \chi_{\beta}, \gamma\right), \\
& \Psi_{+,-1,1,-1,-1,0}^{+}\left(r_{\alpha}, \chi_{\alpha}, r_{\beta}, \chi_{\beta} \gamma\right), \\
& \Psi_{+, 0,2,0,0,0}^{+}\left(r_{\alpha}, \chi_{\alpha}, r_{\beta}, \chi_{\beta}, \gamma\right), \\
& \Psi_{+,-2,0,0,0,0}^{+}\left(r_{\alpha}, \chi_{\alpha}, r_{\beta}, \chi_{\beta}, \gamma\right), \\
& \Psi_{+, 0,2,-2,-2,0}^{+}\left(r_{\alpha}, \chi_{\alpha}, r_{\beta}, \chi_{\beta}, \gamma\right), \\
& \Psi_{+,-2,0,2,2,0}^{+}\left(r_{\alpha}, \chi_{\alpha}, r_{\beta}, \chi_{\beta}, \gamma\right), \\
& \Psi_{-, 0,0,-2,0,0}^{+}\left(r_{\alpha}, \chi_{\alpha}, r_{\beta}, \chi_{\beta}, \gamma\right), \\
& \Psi_{-, 0,0,0,2,0}^{+}\left(r_{\alpha}, \chi_{\alpha}, r_{\beta}, \chi_{\beta}, \gamma\right), \\
& \Psi_{-, 1,1,-1,1,0}^{+}\left(r_{\alpha}, \chi_{\alpha}, r_{\beta}, \chi_{\beta}, \gamma\right), \\
& \Psi_{-,-1,-1,-1,1,0}^{+}\left(r_{\alpha}, \chi_{\alpha}, r_{\beta}, \chi_{\beta}, \gamma\right), \\
& \Psi_{-, 2,2,-2,0,0}^{+}\left(r_{\alpha}, \chi_{\alpha}, r_{\beta}, \chi_{\beta}, \gamma\right), \\
& \Psi_{-,-2,-2,0,2,0}^{+}\left(r_{\alpha}, \chi_{\alpha}, r_{\beta}, \chi_{\beta}, \gamma\right) .
\end{aligned}
$$

States with $d$-wave symmetry correspond to

$$
\begin{aligned}
& \Psi_{+, 0,2,1,1,0}^{+}\left(r_{\alpha}, \chi_{\alpha}, r_{\beta}, \chi_{\beta}, \gamma\right), \\
& \Psi_{+,-2,0,-1,-1,0}^{+}\left(r_{\alpha}, \chi_{\alpha}, r_{\beta}, \chi_{\beta}, \gamma\right), \\
& \Psi_{+,-1,1,2,2,0}^{+}\left(r_{\alpha}, \chi_{\alpha}, r_{\beta}, \chi_{\beta}, \gamma\right), \\
& \Psi_{+,-1,1,-2,-2,0}^{+}\left(r_{\alpha}, \chi_{\alpha}, r_{\beta}, \chi_{\beta}, \gamma\right), \\
& \Psi_{-, 1,1,0,2,0}^{+}\left(r_{\alpha}, \chi_{\alpha}, r_{\beta}, \chi_{\beta}, \gamma\right), \\
& \Psi_{-,-1,-1,-2,0,0}^{+}\left(r_{\alpha}, \chi_{\alpha}, r_{\beta}, \chi_{\beta}, \gamma\right), \\
& \Psi_{-, 2,2,-1,1,0}^{+}\left(r_{\alpha}, \chi_{\alpha}, r_{\beta}, \chi_{\beta}, \gamma\right), \\
& \Psi_{-,-2,-2,-1,1,0}^{+}\left(r_{\alpha}, \chi_{\alpha}, r_{\beta}, \chi_{\beta}, \gamma\right)
\end{aligned}
$$

Finally, states with $f$-wave symmetry read

$$
\begin{aligned}
& \Psi_{+, 0,2,2,2,0}^{+}\left(r_{\alpha}, \chi_{\alpha}, r_{\beta}, \chi_{\beta}, \gamma\right), \\
& \Psi_{+,-2,0,-2,-2,0}^{+}\left(r_{\alpha}, \chi_{\alpha}, r_{\beta}, \chi_{\beta}, \gamma\right), \\
& \Psi_{-, 2,2,0,2,0}^{+}\left(r_{\alpha}, \chi_{\alpha}, r_{\beta}, \chi_{\beta}, \gamma\right), \\
& \Psi_{-,-2,-2,-2,0,0}^{+}\left(r_{\alpha}, \chi_{\alpha}, r_{\beta}, \chi_{\beta}, \gamma\right) .
\end{aligned}
$$

As far as the other linear combinations

$$
\Psi_{\sigma, m_{+}^{\alpha}, m_{-}^{\alpha}, m_{+}^{\beta}, m_{-}^{\beta}, m}^{-}\left(r_{\alpha}, \chi_{\alpha}, r_{\beta}, \chi_{\beta}, \gamma\right)
$$

are concerned, due to the extra minus sign under the transformation $O^{\prime}$ in Eq. (B.8), the above classification is still valid, provided one makes the replacements $s \Longleftrightarrow f$ and $p \Longleftrightarrow d$.

Note that the $f$-wave states differ in their transformation properties under $R$ with respect to the magnon-mediated two-hole ground states $\Psi(r, \varphi)$ which are invariant 
under $R$. Accordingly, in contrast to the square lattice case, there is no one-to-one correspondence between these magnon-mediated two-hole bound states and the $f$-wave states formed by two holes localized on a Skyrmion. Still, one of the candidate twohole-Skyrmion ground states, and one of the candidate two-hole-anti-Skyrmion ground states (i.e., those with smallest quantum numbers) correspond to the wave functions $\Psi_{+,-1,1,0,0,0}^{-}\left(r_{\alpha}, \chi_{\alpha}, r_{\beta}, \chi_{\beta}, \gamma\right)$ and $\Psi_{-, 0,0,-1,1,0}^{-}\left(r_{\alpha}, \chi_{\alpha}, r_{\beta}, \chi_{\beta}, \gamma\right)$, which both have $f$-wave symmetry. It should be pointed out again that the pairing symmetry in the dehydrated version of $\mathrm{Na}_{2} \mathrm{CoO}_{2} \times y \mathrm{H}_{2} \mathrm{O}$ - an experimental realization of a hole-doped honeycomb lattice antiferromagnet — indeed appears to be $f$-wave [32].

\section{References}

[1] C. Brügger, F. Kämpfer, M. Moser, M. Pepe, and U.-J. Wiese, Phys. Rev. B 74, $224432(2006)$.

[2] F. Kämpfer, B. Bessire, M. Wirz, C. P. Hofmann, F.-J. Jiang, and U.-J. Wiese, Phys. Rev. B 85, 075123 (2012).

[3] J. Gasser, M. E. Sainio, and A. Svarc, Nucl. Phys. B 307, 779 (1988).

[4] E. Jenkins and A. Manohar, Phys. Lett. B 255, 558 (1991).

[5] V. Bernard, N. Kaiser, J. Kambor, and U.-G. Meissner, Nucl. Phys. B 388, 315 (1992).

[6] T. Becher and H. Leutwyler, Eur. Phys. J. C 9, 643 (1999).

[7] F. Kämpfer, M. Moser, and U.-J. Wiese, Nucl. Phys. B 729, 317 (2005).

[8] C. Brügger, F. Kämpfer, M. Pepe, and U.-J. Wiese, Eur. Phys. J. B 53, 433 (2006).

[9] C. Brügger, C. P. Hofmann, F. Kämpfer, M. Pepe, and U.-J. Wiese, Phys. Rev. B 75, 014421 (2007).

[10] C. Brügger, C. P. Hofmann, F. Kämpfer, M. Moser, M. Pepe, and U.-J. Wiese, Phys. Rev. B 75, 214405 (2007).

[11] F.-J. Jiang, F. Kämpfer, C. P. Hofmann, and U.-J. Wiese, Eur. Phys. J. B 69, 473 (2009).

[12] N. D. Vlasii, C. P. Hofmann, F.-J. Jiang, and U.-J. Wiese, Phys. Rev. B 86, 155113 (2012).

[13] F. D. M. Haldane, Phys. Rev. Lett. 61, 1029 (1988). 
[14] N. Read and S. Sachdev, Phys. Rev. Lett. 62, 1694 (1989); Nucl. Phys. B 316, 609 (1989).

[15] B. I. Shraiman and E. D. Siggia, Phys. Rev. B 42, 2485 (1990).

[16] R. J. Gooding, Phys. Rev. Lett. 66, 2266 (1991).

[17] R. J. Gooding and A. Mailhot, Phys. Rev. B 48, 6132 (1993).

[18] S. Haas, F.-C. Zhang, F. Mila, and T. M. Rice, Phys. Rev. Lett. 77, 3021 (1996).

[19] E. C. Marino and M. B. Silva Neto, Phys. Rev. B 64, 092511 (2001).

[20] O. I. Motrunich and A. Vishwanath, Phys. Rev. B 70, 075104 (2004).

[21] T. Sentil, A. Vishwanath, L. Balents, S. Sachdev, and M. P. A. Fisher, Science 303, 1490 (2004); Phys. Rev. B 70, 144407 (2004).

[22] O. Bär, M. Imboden, and U.-J. Wiese, Nucl. Phys. B 686, 347 (2004).

[23] U.-J. Wiese, Nucl. Phys. Proc. Suppl. 141, 143 (2005).

[24] T. Morinari, Phys. Rev. B 72, 104502 (2005).

[25] L. Fu, S. Sachdev, and C. Xu, Phys. Rev. B 83, 165123 (2011).

[26] I. Raicevic, D. Popovic, C. Panagopoulos, L. Benfatto, M. B. Silva Neto, E. S. Choi, and T. Sasagawa, Phys. Rev. Lett. 106, 227206 (2011).

[27] G. Baskaran, arXiv:1108.3562.

[28] J. A. Vergés, E. Louis, P. S. Lohmdahl, F. Guinea, and A. R. Bishop, Phys. Rev. B 43, 6099 (1991).

[29] G. Seibold, Phys. Rev. B 58, 15520 (1998).

[30] M. Berciu and S. John, Phys. Rev. B 59, 15143 (1999).

[31] C. Timm and K. H. Bennemann, Phys. Rev. Lett. 84, 4994 (2000).

[32] I. I. Mazin and M. D. Johannes, Nat. Phys. 1, 91 (2005).

[33] C. Honercamp, Phys. Rev. Lett. 100, 146404 (2008).

[34] W.-C. Lee, C. Wu, and S. Das Sarma, Phys. Rev. A 82, 053611 (2010).

[35] R. Ganesh, S. Nishimoto, and J. van den Brink, Phys. Rev. B 87, 054413 (2013).

[36] X. G. Wen and A. Zee, Phys. Rev. Lett. 61, 1025 (1988).

[37] T. Dombre and N. Read, Phys. Rev. B 38, 7181 (1988). 
[38] E. Fradkin and M. Stone, Phys. Rev. B 38, 7215 (1988).

[39] F.-J. Jiang, F. Kämpfer, M. Nyfeler, and U.-J. Wiese, Phys. Rev. B 78, 214406 (2008). 